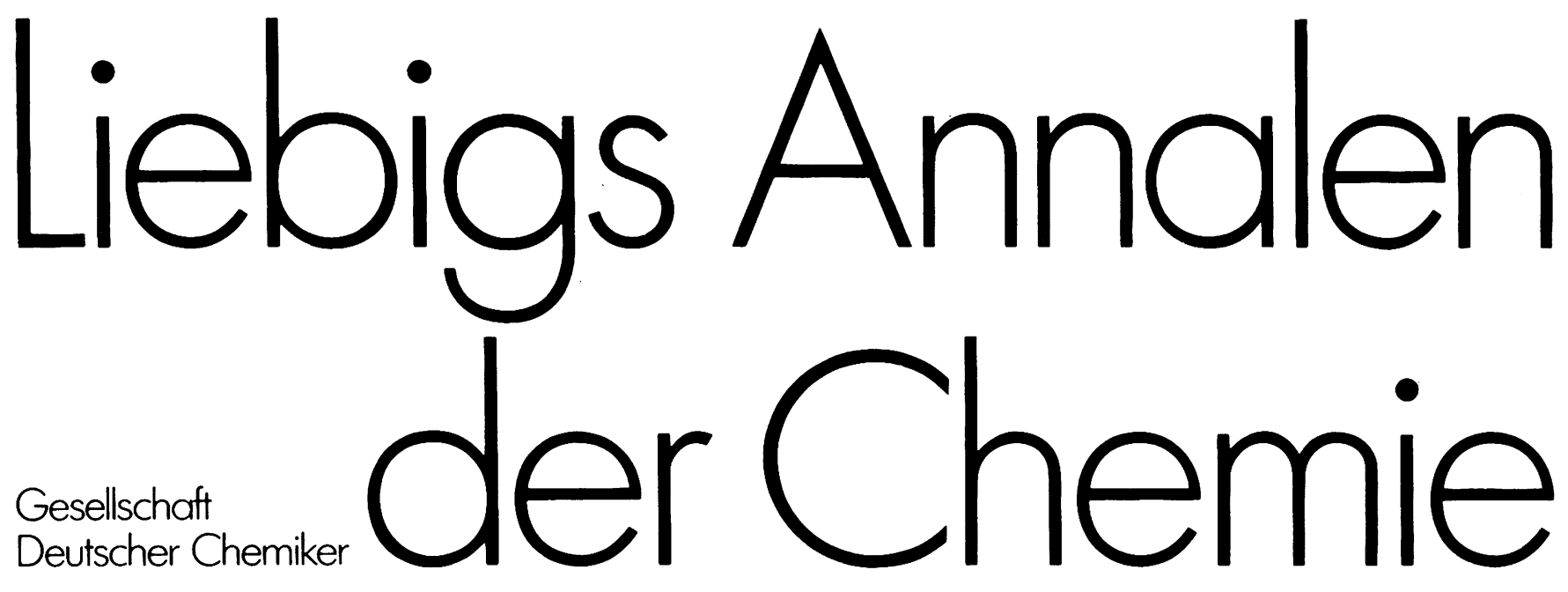

\title{
1993
}

Editorial Board: $\quad$ W. Francke $\cdot$ K. Hafner $\cdot$ H. Hopf $\cdot$ H. Kessler $\cdot$ J. Mulzer $\cdot$ H. Paulsen $\cdot$ E. Schmitz $\cdot$ K. Schreiber $\cdot$ H. A. Staab

$\begin{array}{ll}\text { Managing Editor: } & \text { R. Temme } \\ \text { Associate Editor: } & \text { W. Jung } \\ \text { Administration: } & \text { H. Holm } \\ \text { Production: } & \text { O. Olivecká }\end{array}$

Editorial Office: $\quad$ Liebigs Annalen der Chemie

P.O. Box 101161

D-69451 Weinheim

Federal Republic of Germany

Telephone (internat.) +496201606-255

Telefax (internat.) $\quad+496201606-328$
Publisher: $\quad \mathrm{VCH}$ Verlagsgesellschaft $\mathrm{mbH}$

P.O. Box 101161

D-69451 Weinheim

Federal Republic of Germany

Telephone (internat.) +496201 606-0

Telefax (internat.) +49 6201606-328

Telex

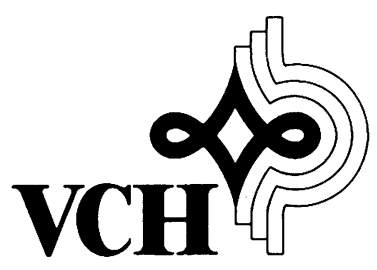




\title{
Asymmetrische Synthesen mit chiralen 1,4-Oxazin-2,5-dionen: Darstellung enantiomerenreiner 2-substituierter Pipecolinsäurederivate ${ }^{\text {h/ }}$
}

\author{
Klaus Th. Wanner* und Stamatios Stamenitis \\ Institut für Pharmazie der Freien Universität Berlin, \\ Königin-Luise-Straße $2+4,1000$ Berlin 33
}

Eingegangen am 30. November 1992

Key Words: 1,4-Oxazines, chiral/Pipecolic acid derivatives / Amino acids / 2-Piperidinecarboxylic acid derivatives

Asymmetric Synthesis with Chiral 1,4-Oxazine-2,5-diones: Preparation of Enantiomerically Pure 2-Substituted Pipecolic Acid Derivatives

A new asymmetric synthesis of $\alpha$-amino acids is presented. This synthesis is based on the chiral 1,4-oxazine-2,5-diones 5 and 14 relying on the $\alpha$-hydroxy acid 12 as a chiral auxiliary. A base-mediated alkylation of these chiral amino acid building blocks $(5,12)$ with different alkyl halides proceeds, after deprotonation with sec-butyllithium, with high yields and excellent d.s. (up to 99.5/0.5). As exemplified by the synthesis of $\mathbf{1 5}$ (in comparison to that of $\mathbf{7 a}$ ) the absolute configu- ration of the stereocenter in the amino acid unit is determined by the sequence the substituents are introduced. In the enolate $\mathbf{6}$ and in that of $\mathbf{1 4}$ the electrophile adds consistently to the re face of the prochiral carbon. From the alkylation products the corresponding 2-substituted pipecolic acid derivatives are obtained in good yields upon hydrolysis under basic or acidic conditions.
Die asymmetrische Synthese von $\alpha$-Aminosäuren ist ein Gebiet, das zur Zeit sehr lebhaft bearbeitet wird. Neben den seltenen, nichtproteinogenen Aminosäuren sind es vor allem die synthetischen, nicht natürlich vorkommenden Derivate und hier insbesondere die $\alpha$-disubstituierten $\alpha$-Aminosäuren, denen das Hauptaugenmerk gilt. Von den natürlichen $\alpha$-Aminosäuren unterscheiden sich die $\alpha$-disubstituierten Derivate durch ihre stark veränderten, häufig interessanten, pharmakologischen und biochemischen Eigenschaften; so können sie beispielsweise als Enzyminhibitoren fungieren. In der Arzneimittelforschung werden sie auch häufig anstelle von natürlichen Aminosäuren als Bausteine für physiologisch aktive Peptide verwendet. Das Ziel ist dabei eine Verbesserung der Stabilität und gegebenenfalls auch Wirkungsqualität ${ }^{[1]}$.

Für die asymmetrische Synthese $\alpha, \alpha$-disubstituierter Aminosäuren sind seit längerem mehrere Verfahren ${ }^{[2]}$ bekannt, wobei die Zahl der Methoden zur Darstellung $\alpha$ substituierter cyclischer Aminosäuren ${ }^{[3]}$ - wie z.B. des Prolins oder der Pipecolinsäure - allerdings noch begrenzt ist. Die Mehrzahl der asymmetrischen Aminosäuresynthesen beruhen im Prinzip auf der stereoselektiven Alkylierung von chiralen Glycinäquivalenten, bestes Beispiel ist die bekannte und bewährte Bislactimethermethode von Schöllkopf. In Abwandlung dieses Verfahrens wurden von Schöllkopf et al. auch asymmetrische Synthesen mit Oxazinonen II vorgestellt ${ }^{[4]}$.

Im Zusammenhang mit dieser Methode erscheint es naheliegend, als Ansatz für eine asymmetrische Aminosäuresynthese 1,4-Oxazindione (vom Typ I) zu verwenden. Solche Verfahren sind unseres Wissens aber bisher nicht beschrieben worden, vermutlich aus Mangel an geeigneten, konfigurationsstabilen (möglichst nicht enolisierbaren) und

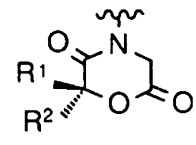

I<smiles>[R7]C1([2H])OC(=O)CN=C1O</smiles>

II enantiomerenreinen $\alpha$-Hydroxycarbonsäuren, die die chirale Hilfsgruppe bilden. Wir stellen hier nun ein Verfahren zur asymmetrischen Synthese $\alpha$-substituierter Pipecolinsäure-Derivate vor, das auf dem eben genannten Prinzip beruht. Als chirale und konfigurationsstabile Hilfsgruppe dient uns dabei die $\alpha$-Hydroxycarbonsäure 12, die in zwei Reaktionsschritten aus käuflicher Camphansäure darstellbar ist und die wir schon früher in anderem Zusammenhang verwendeten ${ }^{[5]}$.

Als Ausgangsverbindung für die Darstellung des 1,4Oxazin-2,5-dions 5 - als Baustein für asymmetrische Synthesen $\alpha$-substituierter Pipecolinsäure-Derivate - wurde auf die Carbonsäure $\mathbf{1}^{[5]}$, ein $O$-Benzyl-Derivat der $\alpha$-Hydroxycarbonsäure 12 zurückgegriffen.

Die Synthese von 5 ließ sich wie im Schema 1 angegeben realisieren, indem zuerst die Amidbindung geknüpft $\left[(\mathrm{COCl})_{2}\right.$, Pipecolinsäureethylester $\left.\rightarrow 3\right]$ und dann nach hydrogenolytischer Benzylgruppenabspaltung $\left(\mathrm{H}_{2}, \mathrm{Pd} / \mathrm{C} \rightarrow 4\right)$ durch basische Umesterung (1.05 Äquiv. $n$-BuLi, $-78^{\circ} \mathrm{C}$, THF) die Lactonbindung geschlossen wurde $(\rightarrow 5)$. Die Verbindungen 3, 4 und 5 fielen dabei infolge der racemischen Natur von 2 als Diastereomerengemische an (ca. 1:1) und wurden in dieser Form jeweils weiterverwendet. Die Ausbeuten betrugen 94\% (3), 85\% (4) und 97\% (5). Die Strukturen wurden für 3 und 4 anhand der analytischen 
Schema 1

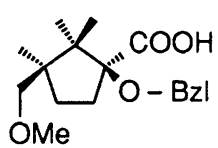

1
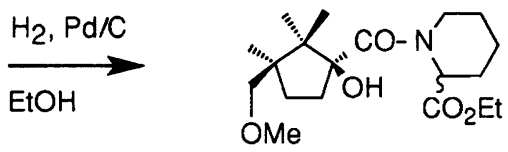

4

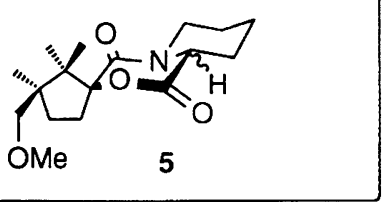

Daten der Isomerengemische gesichert. Bei 5 wurden hierzu die durch Säulenchromatographie zugänglichen, isomerenreinen Verbindungen verwandt. Der Ringschluß zu 5 wird dabei insbesondere im IR-Spektrum durch das Auftreten einer Lacton-Carbonylschwingung (ca. $1744 \mathrm{~cm}^{-1}$ ) in Verbindung mit der im ${ }^{1} \mathrm{H}$-NMR-Spektrum fehlenden Ethylestereinheit deutlich.

Die Alkylierung des 1,4-Oxazindions 5 erwies sich als völlig unproblematisch. Wir führten sie nach folgendem Schema durch: Zunächst wurde mit $s$-Butyllithium in THF bei $-78^{\circ} \mathrm{C}$ deprotoniert, dann nach 30 min das Alkylierungsagenz zugegeben und das Reaktionsgemisch anschlieBend auf Raumtemperatur erwärmt. Durch die Temperaturerhöhung wurde sichergestellt, daß die Reaktion nahezu quantitativ verlief; ein erkennbarer Verlust an Stereoselektivität war damit nicht verbunden. So wurde beispielsweise bei der Reaktion mit Benzylbromid nach wäßriger Aufarbeitung und SC 7a mit einer Ausbeute von 93\% in isomerenreiner Form erhalten. Vergleichbar gute Resultate wurden bei der Umsetzung mit Methyliodid (7b, Ausb. 97\%) und Allylbromid (7c, Ausb. 97\%) erzielt ${ }^{[6]} .5$ wurde dabei immer als Diastereomerengemisch eingesetzt. Zur Kontrolle wurde die Alkylierung mit Benzylbromid außerdem auch mit isomerenreinem 5 durchgeführt. Wie zu erwarten, zeigten sich dabei aber keine Unterschiede. Trotz eingehender Untersuchungen mittels ${ }^{1} \mathrm{H}-\mathrm{NMR}$-Spektroskopie sowie Hochdruckflüssigkeitschromatographie ließ sich in keinem der Reaktionsprodukte ein Nebenisomer nachweisen. Dies war bereits ein erster Hinweis darauf, daß die Alkylierungen von 5 hochstereoselektiv verlaufen waren. Durch HPLC-Analyse konnte dies schließlich auch bewiesen werden, nachdem das zu 7a epimere Alkylierungsprodukt 15 als Referenzsubstanz durch eine andere Synthese (siehe unten) zur Verfügung stand. Die liquidchromatographische Analyse ergab für die Diastereoselektivität (für die Reaktion zu 7a) einen Wert von mindestens 99.5/0.5 (7a/15), das Nebenisomer 15 war dabei kaum mehr wahrnehmbar. Auch bei den Reaktionen zu $\mathbf{7 b}$ und $\mathbf{7} \mathbf{c}$ war die asymmetrische Induktion sehr gut; die Bestimmung wurde aber auf einem anderen Weg vorgenommen (siehe unten).

Durch Erhitzen mit Salzsäure $\left(160^{\circ} \mathrm{C}, 4 \mathrm{~h}, 7 \mathbf{a}\right.$ und $\left.7 \mathbf{b}\right)$ oder mit $\mathrm{LiOH}$ in Ethylenglycoldimethylether $\left(160^{\circ} \mathrm{C}, 48 \mathrm{~h}\right.$, 7c) und anschließende chromatographische Reinigung an einem sauren Ionenaustauscher konnten aus den Alkylierungsprodukten die zugrundeliegenden Aminosäuren $\mathbf{8 a}-\mathbf{c}$ mit Ausbeuten von 80, 76 und $64 \%$ erhalten werden (Schema 2). Die absolute Konfiguration der Aminosäure (S)-8b folgt aus ihrem optischen Drehwert durch Vergleich mit dem Literaturwert $\left([\alpha]_{\mathrm{D}}=-3.7\right.$, Lit. $\left.{ }^{[7]}:[\alpha]_{\mathrm{D}}^{23}=-3.7\right)$. Den Verbindungen $(R)-8 \mathbf{a}$ und $(R)-8 \mathbf{c}$ wurde in Analogie $(R)$-Konfiguration zugeordnet.

Der Konfiguration von $\mathbf{7 b}$ entsprechend wird das Lithiumenolat 6 bevorzugt von der re-Seite („Unterseite“) angegriffen. Worauf dies beruht ist noch unklar. Es erscheint aber naheliegend, da $\beta$ der Angriff immer aus derselben Richtung erfolgt, selbst bei verschiedenen Erstsubstituenten (am $\alpha$-C der Aminosäure). Dies hieße, daß einzig durch die

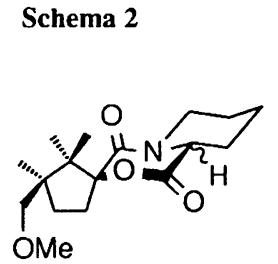

5

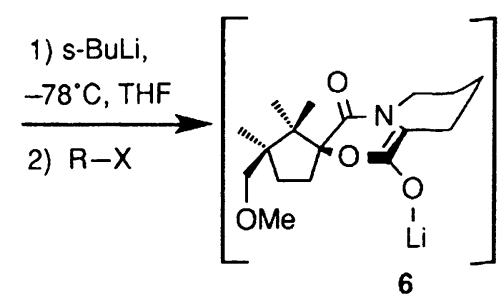

6

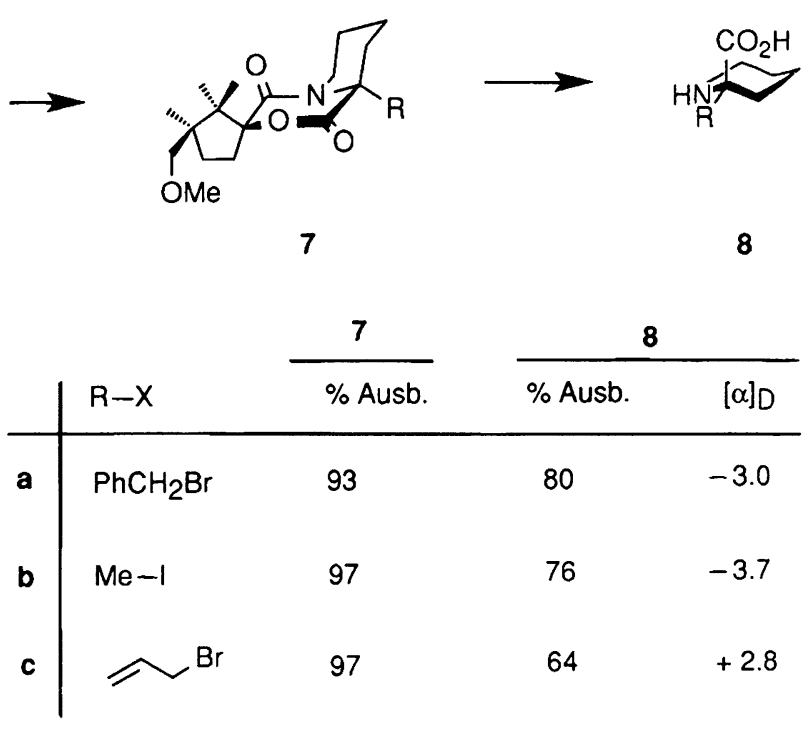

Reihenfolge der Substituenteneinführung die Konfiguration festgelegt und danach mit ein und derselben Hilfsgruppe beide diastereomeren Formen der Alkylierungsprodukte ( 7 bzw. 15) und schließlich beide enantiomeren Formen der 
Aminosäuren zugänglich sein sollten. Zur Überprüfung dieser Annahme wurde das 1,4-Oxazindion 14 synthetisiert.

Schema 3
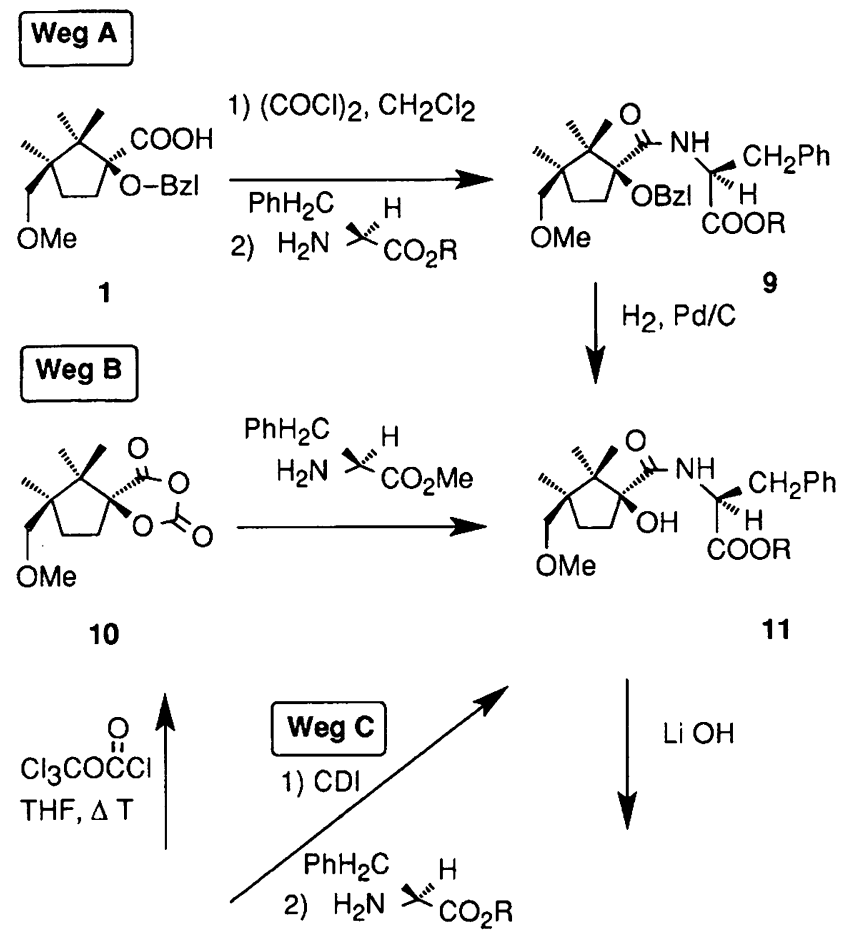

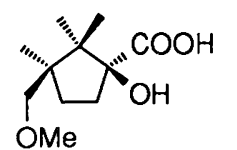

$\mathrm{THF}, \Delta \mathrm{T}$

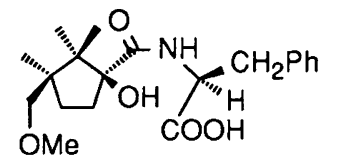

12

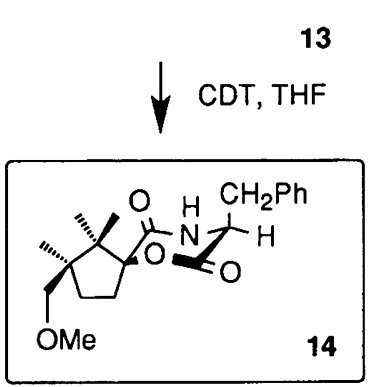

\% Ausb.
\begin{tabular}{lll|l|lllllll}
10 & 14 & & & R & 9 & Weg & A & B & C & 13 \\
\hline \multirow{2}{*}{56} & 72 & a & Me & 87 & & 95 & - & 85 & $78^{[\mathrm{a}]}$ \\
& & b & Et & 93 & & 97 & 59 & $84^{[b]}$ & $94^{[b]}$
\end{tabular}

[a] Aus 11a. - ${ }^{[b]}$ Aus 11b.

Die Synthese von 14 führte über die Verbindungen 11, die auf drei verschiedenen Wegen (A, B und C) dargestellt werden konnten, deren Unterschied hauptsächlich in der Aktivierung der Säurefunktion der chiralen Hilfsgruppe besteht. So wurden die Methyl- und Ethylester 11a und 11b einmal (Weg A) in enger Anlehnung an die Synthese von 4 über das entsprechende Säurechlorid von 1 dargestellt $(\mathbf{1} \rightarrow$ $9 \rightarrow 11$ ). Als Reaktionspartner wurden dabei, wie auch in den folgenden Beispielen zur Vermeidung von Diastereome- rengemischen die entsprechenden L-Phenylalaninester eingesetzt. Bei den beiden anderen Wegen (B und C) wurde von 12 ausgegangen. Diese sind wesentlich vorteilhafter, da dabei die zwei Synthesestufen entfallen, die sonst für die Umwandlung von 12 in 1 erforderlich sind ${ }^{[5]}$. Hinzu kommt, daß sich 12 sehr einfach in die Ester 11a und b umwandeln ließ. Durch Reaktion mit Diphosgen konnte aus 12 das Dioxolan 10 erhalten werden, das nach Aminolyse (nur für 11a durchgeführt) 11 ergab (Weg B). Als Alternative hierzu wurde gefunden, $\mathrm{da} \beta$ sich die Edukte (12 und die Phenylalaninester) unter dem Einfluß von 1,1,'-Carbonyldiimidazol (CDI) auch direkt zu 11 kuppeln lassen (Weg C). Die Ausbeuten dieser Reaktionen waren durchweg zufriedenstellend bis sehr gut (siehe Tabelle in Schema 3). Versuche, die beiden letztgenannten Methoden (Weg B und C) auch für die Darstellung von $\mathbf{4}$ heranzuziehen, zeigten die Grenzen dieser Verfahren auf. So wurde bei der Umsetzung von 10 mit 2 anstelle des gewünschten Säureamids 4 ein Carbamat (von 12) als Aminolyseprodukt erhalten, und beim Versuch 4 analog Weg $C$ darzustellen, war eine Produktbildung (aus 12 und 2) nicht zu beobachten.

Im Unterschied zur Synthese von 5 (aus 4), ließ sich weder der Methyl- noch der Ethylester 11a bzw. 11b unter Basenkatalyse zum 1,4-Oxazindion 14 cyclisieren. Deshalb wurde 11 zur Carbonsäure 13 hydrolysiert, die sich dann durch Aktivierung mit 1,1'-Carbonyldi(1,2,4-triazol) (CDT) zum gewünschten 1,4-Oxazindion 14 überführen ließ.

\section{Schema 4}

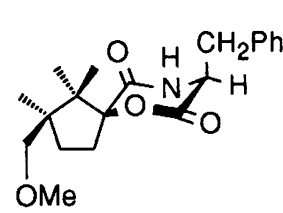

14

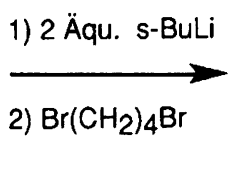

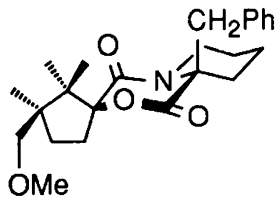

15
Der letzte Syntheseschritt galt dem Aufbau des Piperidinrings und lie $\beta$ sich durch Deprotonierung von 14 mit 2 Äquivalenten $s$-BuLi $\left(-78^{\circ} \mathrm{C}, 2 \mathrm{~h}\right.$ und $1 \mathrm{~h}$ bei $\left.0^{\circ} \mathrm{C}\right)$ sowie anschließende Reaktion mit 1,4-Dibrombutan realisieren (Schema 4) und führte zu der gesuchten, zu 7a diastereomeren Verbindung 15. Wenn auch die Ausbeute nur 32\% betrug, so war die Diastereoselektivität dafür mit mindestens 97.5/2.5 (15/7a) doch sehr gut.

\section{Diastereoselektivitätsbestimmungen}

$\mathrm{Da}$ für eine direkte Bestimmung der asymmetrischen Induktion in der Alkylierungsreaktion $(6 \rightarrow 7)$ die Nebenisomere fehlten - ausgenommen bei 7a mit der Synthese von 15 - wurde die Stereoselektivität über die Enantiomerenreinheit der Aminosäuren 8a-c bestimmt. Das Benzylderivat 8a wurde dabei zur Kontrolle miteingeschlossen.

Dazu wurden die bei der Alkylierung erhaltenen Rohprodukte (!) von 7a-c ohne vorherige Reinigung (im Unterschied dazu beziehen sich die für 7 und 8 oben angegebenen 


\section{Schema 5}

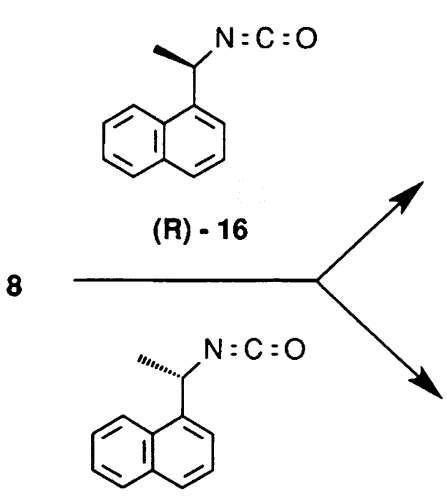

(S) -16

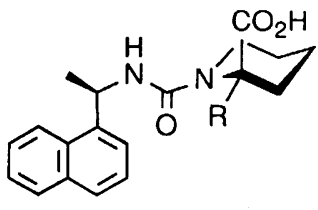

17

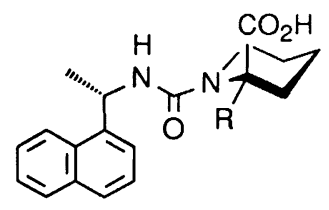

18

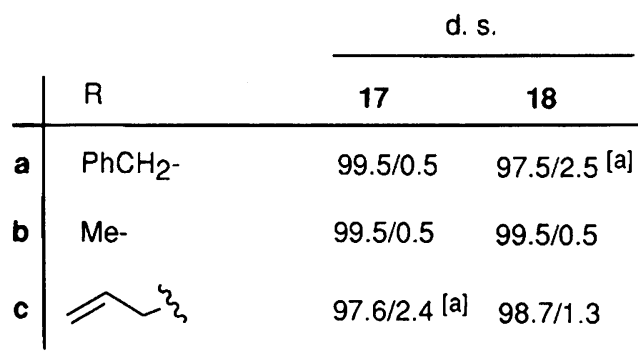

[a] Nebenisomeranteil durch Verunreinigung erhöht.

Ausbeuten auf die reinen Produkte) hydrolysiert, daraus die Aminosäuren 8a-c gewonnen und nach einem Verfahren von Dunlop und Neidle ${ }^{[8]}$ durch Reaktion mit $(R)-1-(1-$ Naphthyl)ethylisocyanat $(R)-16$ und mit $(S)$-1-(1-Naphthyl)ethylisocyanat $(S)-16$ in die diastereomeren Harnstoffe 17 bzw. 18 übergeführt. Die Diastereomerenverhältnisse von 17 (Haupt-/Nebenisomer) und 18 wurden durch HPLC-Analyse an Kieselgel bestimmt, wobei als Referenzsubstanz für das Nebenisomer (zur Bestimmung der Retentionszeit) bei 17 die Verbindung 18 und bei 18 entsprechend Derivat 17 herangezogen wurde. (Das Nebenisomer von 17 ist das Spiegelbild zu 18 und weist deshalb an achiraler Phase dieselbe Retentionszeit wie dieses auf.) Die Ergebnisse der Bestimmungen sind in Schema 5 zusammengestellt. Die Werte für die Nebenisomere lagen bei $17 \mathrm{zwi}$ schen 0.5 und $2.4 \%$ und bei 18 zwischen 0.5 und $2.5 \%$. Durch die geringe Intensität der Nebenisomere und wegen der zum Teil unvollständigen Basislinientrennung waren die Bestimmungen gewissen Schwankungen unterworfen und wurden deshalb mehrfach wiederholt. In die Tabelle wurden die jeweils ungünstigeren Verhältnisse aufgenommen. $\mathrm{Da} ß$ zwischen einzelnen Wertepaaren (17 und 18) Abweichungen bestehen, führen wir auf das Vorliegen von Verunreinigungen zurück (zufällige Erhöhung des Nebenisomeranteils). Die Diastereoselektivität des Alkylierungsschrittes liegt deshalb bei wenigstens $98.7 / 1.3$ für $7 \mathbf{c}$ und bei $99.5 / 0.5$ für $7 \mathbf{a}$ und $7 \mathbf{b}$.

Damit konnte mit dem von der $\alpha$-Hydroxycarbonsäure 12 abgeleiteten 1,4-Oxazindion 5 ein Reagenz zur hochste-

reoselektiven Synthese von $\alpha$-substituierten Pipecolinsäurederivaten entwickelt werden. Untersuchungen zur Verallgemeinerung dieses Verfahrens über ein entsprechendes chirales Glycinäquivalent und zum Induktionsmechanismus sind Gegenstand weiterer Untersuchungen.

Wir danken der Deutschen Forschungsgemeinschaft und dem Fonds der Chemischen Industrie für die finanzielle Unterstützung dieser Arbeit und Frau $V$. Bresien für die Hilfe beim Experimentieren.

\section{Experimenteller Teil}

Schmelzpunkte (nicht korrigiert): Apparatur nach Linström. ${ }^{1}$ H-NMR-Spektren: JNM-GX 400 (Jeol) und AC 300 (Bruker), $\delta$ Skala, TMS als interner Standard (Abkürzungen: Pip. = Piperidinringprotonen, $\mathrm{cPent}=$ Cyclopentanringprotonen). $-\mathrm{MS}$ : $\mathrm{SMCH}$ 7 (Varian), MJ25RS (Kratos) und MATCH 7 (Finnigan). - IRSpektren: IR 4300 (Perkin-Elmer), Flüssigkeiten als Film, Feststoffe als $\mathrm{KBr}$-Preßlinge. - CHN-Analysen: CHN-Rapid (Heraeus), Elemental Analyser $340 \mathrm{~B}$ und $340 \mathrm{C}$ (Perkin-Elmer). - SC: Flash-Chromatographie ${ }^{[9]}$. Zentrifugalchromatographie: Chromatotron, Modell $7924 \mathrm{~T}$ (Harrison Research). HPLC-Apparatur: Pumpe L-6200-Intelligent-Pumpe, L-4250-UV-VIS-Detektor, D2500-Chromato-Integrator (Merck-Hitachi). - Optische Drehungen: Lichtelektrisches Polarimeter Zeiss, 0.5-dm-Rohr; 241 MC Polarimeter (Perkin-Elmer). - Schutzgas: Stickstoff. - Hydrierapparatur: Niederdruck-Hydrierapparat (Adolf Kühner AG, Basel).

(2R)- und (2S)-Ethyl-1,1-[(1S,3R)-1-benzyloxy-3-methoxymethyl-2,2,3-trimethyl-1-cyclopentylcarbonyl]-2-piperidincarboxylat (3): $0.379 \mathrm{~g}(1.238 \mathrm{mmol}) \mathbf{1}^{[5]}$ in $2 \mathrm{ml}$ absol. $\mathrm{CH}_{2} \mathrm{Cl}_{2}$ wurden bei Raumtemp. mit $0.317 \mathrm{~g}(2.500 \mathrm{mmol})$ Oxalylchlorid und einem Tropfen Dimethylformamid versetzt und anschließend bis zur Beendigung der Gasentwicklung gerührt (ca. 2 h). Dann wurde i. Vak. eingeengt und das zurückgebliebene, farblose Öl in $2 \mathrm{ml}$ THF gelöst und mit $3.75 \mathrm{mmol}(0.589 \mathrm{~g}) 2$ bei Raumtemp. versetzt. Nach zweistündigem Rühren wurde i.Vak. eingeengt, der Rückstand in $50 \mathrm{ml}$ Diethylether aufgenommen, nacheinander mit $0.2 \mathrm{~N} \mathrm{HCl}$, $0.2 \mathrm{~N} \mathrm{NaOH}($ je $3 \times 20 \mathrm{ml})$ und ges. NaCl-Lösung $(2 \times 20 \mathrm{ml})$ gewaschen, getrocknet $\left(\mathrm{MgSO}_{4}\right)$ und i.Vak. wieder vom Lösungsmittel befreit. Der Rückstand wurde durch SC ( $n$-Hexan/HOAc, $6: 4)$ getrennt; Ausb. $0.520 \mathrm{~g}(94 \%)$ farbloses Öl, $[\alpha]_{546}=+53.0$, $[\alpha]_{578}=+57.0\left(c=0.5\right.$ in $\left.\mathrm{Et}_{2} \mathrm{O}\right) .-\operatorname{IR}($ Film $): \tilde{v}=3080 \mathrm{~cm}^{-1}$, 1735, 1630. - 'H-NMR $\left(\mathrm{CDCl}_{3}, 400 \mathrm{MHz}\right)$ Diastereomerengemisch, Verhältnis $\approx 1 / 1$, Signalintensitäten beziehen sich auf die einzelnen Diastereomere: $\delta=0.88\left(\mathrm{~s}, 3 \mathrm{H}, \mathrm{CH}_{3}\right), 0.96\left(\mathrm{~s}, 3 \mathrm{H}, \mathrm{CH}_{3}\right)$, $0.97\left(\mathrm{~s}, 6 \mathrm{H}, 2 \mathrm{CH}_{3}\right), 1.08\left(\mathrm{~s}, 3 \mathrm{H}, \mathrm{CH}_{3}\right), 1.15\left(\mathrm{~s}, 3 \mathrm{H}, \mathrm{CH}_{3}\right), 1.26(\mathrm{t}$, $\left.J=7.3 \mathrm{~Hz}, 6 \mathrm{H}, 2 \mathrm{OCH}_{2} \mathrm{CH}_{3}\right), 1.33-1.77$ (m, 12H, Pip. und cPent), 1.80-1.87 (m, 2H, cPent), 1.96-2.06 (m, 2H, cPent), 2.17-2.34 (m, 2H, Pip.), 2.82-2.91 (m, 2H, cPent), 3.07 (dt, $J=2.9 / 13 \mathrm{~Hz}$, $\left.1 \mathrm{H}, \mathrm{NHCH}_{\mathrm{ax}}\right), 3.14\left(\mathrm{~d}, J=8.1 \mathrm{~Hz}, 1 \mathrm{H}, \mathrm{CH}_{2} \mathrm{OCH}_{3}\right), 3.16(\mathrm{~d} . J=$ $\left.8.8 \mathrm{~Hz}, 1 \mathrm{H}, \mathrm{CH}_{2} \mathrm{OCH}_{3}\right), 3.19-3.26\left(\mathrm{~m}, 1 \mathrm{H}, \mathrm{NHCH}_{\mathrm{ax}}\right.$, Signal z.T. verdeckt), $3.27\left(\mathrm{~s}, 3 \mathrm{H}, \mathrm{OCH}_{3}\right), 3.30\left(\mathrm{~s}, 3 \mathrm{H}, \mathrm{OCH}_{3}\right), 3.55(\mathrm{~d}, J=$ $\left.8.1 \mathrm{~Hz}, 1 \mathrm{H}, \mathrm{CH}_{2} \mathrm{OCH}_{3}\right), 3.58\left(\mathrm{~d}, \mathrm{~J}=8.8 \mathrm{~Hz}, 1 \mathrm{H}, \mathrm{CH}_{2} \mathrm{OCH}_{3}\right.$ ), $4.16-4.24\left(\mathrm{~m}, 2 \times 2 \mathrm{H}, 2 \mathrm{COOCH}_{2} \mathrm{CH}_{3}\right), 4.39(\mathrm{~d}, J=11.5 \mathrm{~Hz}$, $1 \mathrm{H}, H \mathrm{CHPh}), 4.56\left(\mathrm{~d}, J=11.7 \mathrm{~Hz}, 1 \mathrm{H}, \mathrm{CH}_{2} \mathrm{Ph}\right), 4.61(\mathrm{~d}, J=$ $11.7 \mathrm{~Hz}, \mathrm{CH}_{2} \mathrm{Ph}$ ), 4.66 (d, $j=1 \mathrm{i} .5 \mathrm{~Hz}, \mathrm{i} \mathrm{H}, \mathrm{HCi} \mathrm{Ph}$ ), 4.87 (d, breit, $J=14.9 \mathrm{~Hz}, 1 \mathrm{H}, \mathrm{NHCH}_{\mathrm{eq}}$ ), 4.96 (d, breit, $J=13 \mathrm{~Hz} .1 \mathrm{H}$, $\mathrm{NHCH}_{\text {eq }}$ ), 5.38 (d, breit, $\left.J=4.7 \mathrm{~Hz}, 1 \mathrm{H}, \mathrm{CHCOOEt}\right), 5.56$ (d, breit, $J=4.7 \mathrm{~Hz}, 1 \mathrm{H}, \mathrm{CHCOOEt}), 7.26-7.38$ (m, 10H, arom.). MS (70 eV): $m / z=445\left[\mathrm{M}^{+}\right]$.

$$
\begin{array}{ll}
\mathrm{C}_{26} \mathrm{H}_{39} \mathrm{NO}_{5} \text { (445.6) } & \text { Ber. C } 70.08 \mathrm{H} 8.82 \text { N } 3.14 \\
& \text { Gef. C } 70.25 \mathrm{H} 9.01 \text { N } 2.94
\end{array}
$$


(2R)- und (2S)-Ethyl-1-[(1S,3R)-1-hydroxy-3-methoxymethyl2,2,3-trimethyl-1-cyclopentylcarbonyl]-2-piperidincarboxylat (4): $0.520 \mathrm{~g}(1.160 \mathrm{mmol}) 3 \mathrm{in} 13 \mathrm{ml} \mathrm{EtOH}$ wurden mit $0.100 \mathrm{~g} \mathrm{Pd} / \mathrm{C}$ $(10 \% \mathrm{Pd})$ versetzt und $14 \mathrm{~h}$ bei Normaldruck mit $\mathrm{H}_{2}$ in der Hydrierapparatur gerührt. Anschließend wurde filtriert, i.Vak. eingeengt und durch SC gereinigt ( $n$-Hexan/EtOAc, 6:4), Ausb. $0.353 \mathrm{~g}$ $(85 \%)$ farbloses Öl, $[\alpha]_{546} \approx 0,[\alpha]_{578}=+0.8(c=2.0$ in Ether $)$. IR (Film): $\tilde{v}=3360 \mathrm{~cm}^{-1}, 1740,1630 .-{ }^{1} \mathrm{H}-\mathrm{NMR}\left(\mathrm{CDCl}_{3}, 400\right.$ $\mathrm{MHz}$ ) Diastereomeren- (Relation $\approx 1 / 1$ ) und Rotamerengemisch (Relation $\approx 2 / 1$ ), Signalintensitäten beziehen sich auf die einzelnen Isomere: $\delta=0.82-0.92\left(7 \mathrm{~s}, 21 \mathrm{H}, 7 \mathrm{CH}_{3}\right), 0.98-1.30(5 \mathrm{~s}, 15 \mathrm{H}, 5$ $\left.\mathrm{CH}_{3}\right), 1.36-1.39\left(\mathrm{~m}, 12 \mathrm{H}, 4 \mathrm{OCH}_{2} \mathrm{CH}_{3}\right), 1.48-1.96$ (m, 32H, Pip. und cPent), 2.20-2.35 (m, 3H, Pip.), 2.36-2.45 (m, 1H, Pip.), 2.55 $\left(\mathrm{dt}, J=3.0 / 12.0 \mathrm{~Hz}, 1 \mathrm{H}, \mathrm{NHCH}_{\mathrm{ax}}\right), 2.70-2.87$ (m, 2H, Pip.), 2.92 (dt, $J=3.4 / 12.0 \mathrm{~Hz}, 2 \mathrm{H}, \mathrm{NHCH}_{\mathrm{ax}}$ ), 2.98-3.05 (m, 5H), 3.06-3.14 (m, 1H, Pip.), 3.22-3.38 (m, 8H, cPent und $\left.\mathrm{OCH}_{3}\right), 3.40$ (s, 6H, 2 $\left.\mathrm{OCH}_{3}\right), 3.42\left(\mathrm{~s}, 3 \mathrm{H}, \mathrm{OCH}_{3}\right), 4.15-4.25\left(\mathrm{~m}, 8 \mathrm{H}, \mathrm{OCH}_{2} \mathrm{CH}_{3}\right)$, 4.54-4.60 (m, 2H, Pip.), 4.82-4.85 (m, 1H, Pip.), 5.23 (d, breit, $J=14 \mathrm{~Hz}, 1 \mathrm{H}$, Pip.), 5.25 (s, $1 \mathrm{H}, \mathrm{OH}), 5.37$ (s, $1 \mathrm{H}, \mathrm{OH}), 5.41$ ( $\mathrm{m}_{\mathrm{c}}, 1 \mathrm{H}$, Pip.), 5.46 (d, breit $J=3.0 \mathrm{~Hz}, 1 \mathrm{H}$, Pip.), 5.65 (s, 2H, 2 $\mathrm{OH}), 5.88$ (d, breit, $J=4 \mathrm{~Hz}, 1 \mathrm{H}$, Pip.), 6.12 ( $\mathrm{m}_{\mathrm{c}}, 1 \mathrm{H}$, Pip.). - MS $(70 \mathrm{eV}): m / z=355\left[\mathrm{M}^{+}\right]$.

\section{$\mathrm{C}_{19} \mathrm{H}_{33} \mathrm{NO}_{5}$ (355.5) Ber. C 64.19 H 9.35 N 3.94 Gef. C 63.91 H 9.29 N 4.24}

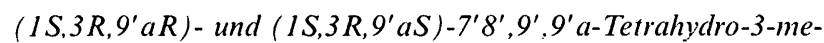
thoxymethyl-2,2,3-trimethylspiro[cyclopentan-1,3' (4' H)-pyrido-

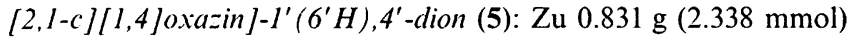
4 in $25 \mathrm{ml}$ absol. THF wurden bei $-78^{\circ} \mathrm{C}$ unter Rühren 2.450 mmol $n$-BuLi $(1.530 \mathrm{ml} 1.6 \mathrm{M}$ Hexanlösung) zugespritzt. Nach zweistündigem Rühren bei $-78^{\circ} \mathrm{C}$ wurde noch $30 \mathrm{~min}$ bei $0^{\circ} \mathrm{C}$ und 30 min bei Raumtemp. gehalten und dann mit $\mathrm{KH}_{2} \mathrm{PO}_{4}-\mathrm{Puffer}$ ( $\mathrm{pH}=4.25$, eisgekühlt) versetzt und i.Vak. vom THF befreit. Anschließend wurde $3 \mathrm{mal}$ mit $30 \mathrm{ml}$ Diethylether ausgeschüttelt und nach Trocknen der Extrakte $\left(\mathrm{MgSO}_{4}\right)$ i.Vak. eingeengt. Aus dem Rückstand wurden durch SC ( $n$-Hexan/EtOAc 7:3) zwei Isomere (Isomer 1 vor $2 \mathrm{im}$ Eluat) erhalten; Gesamtausb. $0.723 \mathrm{~g}(97 \%)$, Isomerenverhältnis Isomer 1/Isomer 2 wie $6 / 4$.

Isomer 1: Farblose Kristalle, Schmp. $78^{\circ} \mathrm{C},[\alpha]_{546}=+22.6,[\alpha]_{576}=$ $+20.1\left(c=0.75\right.$ in Diethylether). - IR (KBr): $\tilde{v}=2920 \mathrm{~cm}^{-1}$, 1744, 1656. - ${ }^{1} \mathrm{H}-\mathrm{NMR}\left(\mathrm{CDCl}_{3}, 400 \mathrm{MHz}\right): \delta=0.90\left(\mathrm{~s}, 3 \mathrm{H}, \mathrm{CH}_{3}\right)$, 0.98 (s, 3H, $\left.\mathrm{CH}_{3}\right), 1.03$ (s, 3H, $\left.\mathrm{CH}_{3}\right), 1.41-1.62$ (m, 3H, Pip.), $1.75-1.78$ (m, 2H, Pip.), 1.90-2.00 (m, 3H, cPent), 2.39-2.42 (m, $1 \mathrm{H}$, Pip.), 2.55 (dt, $\left.J=3.4 / 13 \mathrm{~Hz}, 1 \mathrm{H}, \mathrm{NHCH}_{\mathrm{ax}}\right), 2.77-2.85(\mathrm{~m}$, $1 \mathrm{H}, \mathrm{cPent}), 3.23$ (d, $\left.J=9 \mathrm{~Hz}, 1 \mathrm{H}, \mathrm{HCHOCH}_{3}\right), 3.32(\mathrm{~s}, 3 \mathrm{H}$, $\mathrm{OCH}_{3}$ ), 3.39 (d, $\left.J=9 \mathrm{~Hz}, 1 \mathrm{H}, H \mathrm{CHOCH}_{3}\right), 3.97$ (dd, $J=3.2 /$ $11.7 \mathrm{~Hz}, 1 \mathrm{H}, \mathrm{NC} H), 4.66\left(\mathrm{td}, J=3.6 / 13 \mathrm{~Hz}, 1 \mathrm{H}, \mathrm{NHC}_{\mathrm{eq}}\right)$. - MS $(70 \mathrm{eV}): \mathrm{m} / \mathrm{z}=309\left[\mathrm{M}^{+}\right]$.

\section{$\mathrm{C}_{17} \mathrm{H}_{27} \mathrm{NO}_{4}$ (309.4) Ber. C $65.99 \mathrm{H} 8.78$ N 4.52 Gef. C 66.02 H 8.48 N 4.61}

Isomer 2: Farblose Kristalle, Schmp. $44^{\circ} \mathrm{C},[\alpha]_{546}=+4.2,[\alpha]_{578}=$ $+2.3\left(c=0.5\right.$ in Diethylether). - IR $(\mathrm{KBr}): \tilde{v}=2944 \mathrm{~cm}^{-1}, 1743$, 1656. - ' $\mathrm{H}-\mathrm{NMR}\left(\mathrm{CDCl}_{3}, 400 \mathrm{MHz}\right): \delta=0.96\left(\mathrm{~s}, 3 \mathrm{H}, \mathrm{CH}_{3}\right), 0.97$ (s, 3H, $\left.\mathrm{CH}_{3}\right), 1.04$ (s, 3H, $\left.\mathrm{CH}_{3}\right), 1.38-1.64$ (m, 4H, Pip. und cPent), 1.76-1.80 (m, 1H, cPent), 1.91-2.04 (m, 3H, Pip. und cPent), $2.39-2.42$ (m, 1 H, Pip.), 2.49 (dt, $J=2.9 / 13 \mathrm{~Hz}, 1 \mathrm{H}, \mathrm{NHCH}_{\mathrm{ax}}$ ), $2.72-2.80$ (m, $1 \mathrm{H}, \mathrm{cPent}), 3.22$ (d, $J=8.8 \mathrm{~Hz}, 1 \mathrm{H}, \mathrm{HCHOCH}_{3}$ ), $3.33\left(\mathrm{~s}, 3 \mathrm{H}, \mathrm{OCH}_{3}\right), 3.41\left(\mathrm{~d}, J=8.8 \mathrm{~Hz}, 1 \mathrm{H}, H \mathrm{CHOCH}_{3}\right), 4.03$ (dd, $J=2.9 / 12.0 \mathrm{~Hz}, 1 \mathrm{H}, \mathrm{NCHR}), 4.68(\mathrm{td}, J=2.1 / 13 \mathrm{~Hz}, 1 \mathrm{H}$, $\left.\mathrm{NHCH}_{\mathrm{eq}}\right) .-\mathrm{MS}(70 \mathrm{eV}): m / z=309\left[\mathrm{M}^{+}\right]$.

$$
\begin{array}{ll}
\mathrm{C}_{17} \mathrm{H}_{27} \mathrm{NO}_{4} \text { (309.4) } & \text { Ber. C } 65.99 \text { H } 8.78 \text { N } 4.52 \\
& \text { Gef. C } 65.88 \text { H } 8.80 \text { N } 4.64
\end{array}
$$

Allgemeine Arbeitsvorschrift für Alkylierungen von 5 zu $\mathbf{7 a - c :}$ Eine Lösung von $5(1 \mathrm{mmol})$ in ca. $10 \mathrm{ml}$ absol. THF wird auf $-78^{\circ} \mathrm{C}$ gekühlt, mit $1 \mathrm{mmol} \mathrm{BuLi}(0.71 \mathrm{ml}, 1.4 \mathrm{M}$ in Hexan) und nach $15 \mathrm{~min}$ mit $1.5 \mathrm{mmol}$ Alkylierungsreagenz versetzt. Nach einstündigem Rühren bei $-78^{\circ} \mathrm{C}$, einer weiteren Stunde bei $0^{\circ} \mathrm{C}$ und einer halben Stunde bei Raumtemp. wird durch Zugabe von ca. $5 \mathrm{ml}$ Eis/Wasser hydrolysiert. Anschließend wird THF i.Vak. entfernt und die erhaltene wäßrige Phase $3 \mathrm{mal} \mathrm{mit} 15 \mathrm{ml} \mathrm{Et}_{2} \mathrm{O}$ extrahiert. Das nach Trocknen $\left(\mathrm{MgSO}_{4}\right)$ und Einengen (i.Vak.) erhaltene Rohprodukt wird wie angegeben gereinigt.

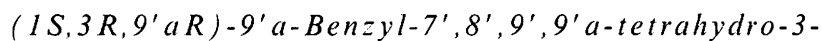
methoxymethyl-2,2,3-trimethylspiro [cyclopentan-1,3' $\left(4^{\prime} H\right)$ pyrido[2,1-c][1,4]-oxazin]-1' $\left(6^{\prime} H\right), 4^{\prime}$-dion (7a) Dargestellt aus $0.300 \mathrm{~g}(0.970 \mathrm{mmol}) 5$ in $6 \mathrm{ml}$ THF mit $1.00 \mathrm{mmol} s$-BuLi und nach $30 \mathrm{~min}$ mit $0.343 \mathrm{~g}(2.000 \mathrm{mmol}, 0.237 \mathrm{ml})$ Benzylbromid. Das Rohprodukt wurde durch SC ( $n$-Hexan/EtOAc, 6:4) gereinigt; Ausb. $0.360 \mathrm{~g}(93 \%)$ farblose Kristalle, Schmp. $93^{\circ} \mathrm{C},[\alpha]_{546}=$ $+22.0,[\alpha]_{578}=+18.2(c=0.5$ in Diethylether $) .-\operatorname{IR}(\mathrm{KBr}): \tilde{v}=$ $3100 \mathrm{~cm}^{-1}, 1740,1660 .-{ }^{1} \mathrm{H}-\mathrm{NMR}\left(\mathrm{CDCl}_{3}, 400 \mathrm{MHz}\right): \delta=$ 0.64-0.72 (m, 1 H, Pip.), 0.80 (s, 3H, $\left.\mathrm{CH}_{3}\right), 0.87$ (s, 3H, $\left.\mathrm{CH}_{3}\right), 0.94$ (s, 3H, $\mathrm{CH}_{3}$ ), $1.21-1.27$ (m, 1H, cPent), 1.38-1.50 (m, 1H, Pip.), $1.54-1.66(\mathrm{~m}, 1 \mathrm{H}, \mathrm{cPent}), 1.82-1.95$ (m, 5H, Pip. und cPent), 2.35-2.42 (m, 1 H, Pip.), 2.80 (d, $J=13.5 \mathrm{~Hz}, 1 \mathrm{H}, \mathrm{HCHPh}$ ), 3.05 $\left(\mathrm{dt}, J=3 / 13.7 \mathrm{~Hz}, 1 \mathrm{H}, \mathrm{NHCH}_{\mathrm{ax}}\right), 3.13(\mathrm{~d}, J=9 \mathrm{~Hz}, 1 \mathrm{H}$, $\left.\mathrm{HCHOCH}_{3}\right), 3.26(\mathrm{~d}, J=9 \mathrm{~Hz}, 1 \mathrm{H}, \mathrm{HCHOCH})_{3}, 3.27(\mathrm{~s}, 3 \mathrm{H}$, $\left.\mathrm{OCH}_{3}\right), 3.67(\mathrm{~d}, J=13.5 \mathrm{~Hz}, 1 \mathrm{H}, H \mathrm{CHPh}), 4.58(\mathrm{td}, J=3.8 /$ $\left.13.7 \mathrm{~Hz}, 1 \mathrm{H}, \mathrm{NHCH}_{\mathrm{cq}}\right), 7.03-7.05(\mathrm{~m}, 2 \mathrm{H}$, arom.), $7.21-7.30(\mathrm{~m}$, $3 \mathrm{H}$, arom. $)$. $\mathrm{MS}(70 \mathrm{eV}): m / z=399\left[\mathrm{M}^{+}\right]$.

$$
\begin{aligned}
& \mathrm{C}_{24} \mathrm{H}_{33} \mathrm{NO}_{4} \text { (399.5) } \quad \text { Ber. C } 72.15 \mathrm{H} 8.32 \text { N } 3.50 \\
& \text { Gef. C } 71.98 \text { H } 8.37 \text { N } 3.51
\end{aligned}
$$

$\left(1 S, 3 R, 9^{\prime} a S\right)-7^{\prime}, 8^{\prime}, 9^{\prime}, 9^{\prime}$ a-Tetrahydro-3-methoxymethyl-2,2,3,9' atetramethylspiro [cyclopentan-1,3' (4'H)-pyrido [2,1-c][1,4]oxazin]-1' $\left(6^{\prime} \mathrm{H}\right), 4^{\prime}$-dion (7b): Dargestellt aus $1.060 \mathrm{~g}(3.430$ mmol) 5 in $35 \mathrm{ml}$ THF mit $3.43 \mathrm{mmol} s$-BuLi und $0.608 \mathrm{~g}(4.28$ mmol, $0.26 \mathrm{ml}) \mathrm{CH}_{3} \mathrm{I}$. Abweichend von der Allgemeinen Arbeitsvorschrift wurde nach Zusatz von Eis/Wasser $(20 \mathrm{ml})$ das THF i. Vak. entfernt, in $100 \mathrm{ml} \mathrm{Et}_{2} \mathrm{O}$ aufgenommen und die organische Phase $3 \mathrm{mal}$ mit $10 \mathrm{ml} 0.05 \mathrm{~N} \mathrm{NaOH}$ und $3 \mathrm{mal}$ mit $10 \mathrm{ml} 0.05 \mathrm{~N} \mathrm{HCl}$ gewaschen. Die organische Phase wurde dann getrocknet $\left(\mathrm{MgSO}_{4}\right)$, i. Vak. vom Lösungsmittel befreit, und der Rückstand wurde durch SC (n-Hexan/EtOAc, 6:4) gereinigt; Ausb. $1.078 \mathrm{~g} \mathrm{(97 \% )} \mathrm{farbloses}$ Öl, $[\alpha]_{546}^{20}=-7.5,[\alpha]_{578}^{20}=-6.78(c=0.8$ in Essigester $)$. - IR (Film: $\tilde{v}=3450 \mathrm{~cm}^{-1}, 1741,1657 .-{ }^{1} \mathrm{H}-\mathrm{NMR}\left(\mathrm{CDCl}_{3}, 300 \mathrm{MHz}\right)$ : $\delta=0.98\left(\mathrm{~s}, 3 \mathrm{H}, \mathrm{CH}_{3}\right), 0.99\left(\mathrm{~s}, 3 \mathrm{H}, \mathrm{CH}_{3}\right), 1.05\left(\mathrm{~s}, 3 \mathrm{H}, \mathrm{CH}_{3}\right)$, 1.25-1.33 (m, 1H, Pip.), 1.33-1.53 (m, 1H, cPent), 1.62 (s, 3H, $\mathrm{NCCH}_{3}$ ), 1.75-1.81 (m, 4H, Pip.), 1.92-2.02 (m, 2H, cPent), 2.17 (d, breit, $J=5.5 \mathrm{~Hz}, 1 \mathrm{H}$, Pip.), 2.67-2.71 (m, 1H, cPent), 2.81 (dt, $\left.J=2.9 / 13.6 \mathrm{~Hz}, 1 \mathrm{H}, \mathrm{NHCH}_{\mathrm{ax}}\right), 3.25(\mathrm{~d}, J=9.0 \mathrm{~Hz}, 1 \mathrm{H}$, $\left.H \mathrm{CHOCH}_{3}\right), 3.37\left(\mathrm{~s}, 3 \mathrm{H}, \mathrm{OCH}_{3}\right), 3.46(\mathrm{~d}, J=9.0 \mathrm{~Hz}, 1 \mathrm{H}$, $\mathrm{HCHOCH}_{3}, 4.46$ (dd, $J=3.2 / 13.6 \mathrm{~Hz}, 1 \mathrm{H}, \mathrm{NHCH}_{\mathrm{eq}}$ ). - MS $(70 \mathrm{eV}): m / z=323\left[\mathrm{M}^{+}\right]$.

$$
\begin{array}{ll}
\mathrm{C}_{18} \mathrm{H}_{29} \mathrm{NO}_{4} \text { (323.4) } & \text { Ber. C } 66.85 \mathrm{H} 9.03 \mathrm{~N} 4.33 \\
& \text { Gef. C } 66.61 \mathrm{H} 9.18 \text { N } 4.09
\end{array}
$$

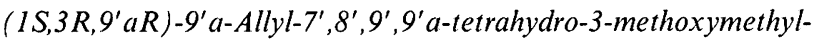
2,2,3-trimethylspiro[cyclopentan-1,3' (4' H)-pyrido [2,1-c][1,4 Joxazin]-1' $\left(6^{\prime} H\right), 4^{\prime}$-dion (7c): Dargestellt aus $0.990 \mathrm{~g}(3.203$ mmol) 5 in $32 \mathrm{ml}$ THF mit $3.363 \mathrm{mmol} \mathrm{s}$-BuLi und nach $20 \mathrm{~min}$ mit $0.582 \mathrm{~g}(4.80 \mathrm{mmol}, 0.407 \mathrm{ml}$ Allylbromid. Abweichend von der Allgemeinen Arbeitsvorschrift wurde nach Zusatz von Eis/Wasser $(20 \mathrm{ml})$ das THF i.Vak. entfernt, der Rückstand in $100 \mathrm{ml} \mathrm{Et}_{2} \mathrm{O}$ aufgenommen, die Etherphase $3 \mathrm{mal}$ mit $10 \mathrm{ml} 0.01 \mathrm{~N} \mathrm{NaOH}$ und 3 mal mit $10 \mathrm{ml} 0.01 \mathrm{~N} \mathrm{HCl}$ gewaschen, getrocknet $\left(\mathrm{MgSO}_{4}\right)$ und 
i. Vak. vom Lösungsmittel befreit. Der Rückstand wurde durch SC ( $n$-Hexan/EtOAc, 7:3) gereinigt; Ausb. $1.084 \mathrm{~g} \mathrm{(97 \% )} \mathrm{farbloses} \mathrm{Öl,}$ $[\alpha]_{545}^{25}=+9.6,[\alpha]_{578}^{25}=+8.1(c=1.0$ in Hexan). IR (Film): $\tilde{v}=$ $2950 \mathrm{~cm}^{-1}, 1730,1650 .{ }^{1} \mathrm{H}-\mathrm{NMR}\left(\mathrm{CDCl}_{3}, 300 \mathrm{MHz}\right): \delta=0.90$ $\left(\mathrm{s}, 3 \mathrm{H}, \mathrm{CH}_{3}\right), 0.95\left(\mathrm{~s}, 3 \mathrm{H}, \mathrm{CH}_{3}\right), 1.04\left(\mathrm{~s}, 3 \mathrm{H}, \mathrm{CH}_{3}\right), 1.40-1.44$ (m, 1H, Pip.), $1.48-1.55$ (m, 1H, cPent.), 1.80-1.99 (m, 6H, Pip. und cPent), 2.22 (d, breit, $J=10.2 \mathrm{~Hz}, 1 \mathrm{H}$, Pip.), 2.37 (dd, $J=7.6 /$ $\left.14.0 \mathrm{~Hz}, 1 \mathrm{H}, \mathrm{HCHCH}=\mathrm{CH}_{2}\right), 2.64-2.78(\mathrm{~m}, 2 \mathrm{H}$, Pip. und cPent), $3.09\left(\mathrm{dd}, J=7.4 / 14.0 \mathrm{~Hz}, 1 \mathrm{H}, \mathrm{HCHCH}=\mathrm{CH}_{2}\right), 3.24(\mathrm{~d}, J=$ $\left.9.0 \mathrm{~Hz}, 1 \mathrm{H}, H \mathrm{CHOCH}_{3}\right), 3.33\left(\mathrm{~s}, 3 \mathrm{H}, \mathrm{OCH}_{3}\right), 3.44(\mathrm{~d}, J=9.0 \mathrm{~Hz}$, $\left.1 \mathrm{H}, \mathrm{HCHOCH}_{3}\right), 4.47$ (dd, $J=4.2 / 13.8 \mathrm{~Hz}, 1 \mathrm{H}, \mathrm{NHCH}_{\mathrm{cq}}$ ), 5.14-5.21 (m, $\left.2 \mathrm{H}, \mathrm{CH}_{2}=\mathrm{CH}\right), 5.21-5.69\left(\mathrm{~m}, 1 \mathrm{H}, \mathrm{HCH}=\mathrm{CH}_{2}\right)$. MS $(70 \mathrm{eV}): \mathrm{m} / z=349\left[\mathrm{M}^{+}\right]$.

$$
\begin{array}{ll}
\mathrm{C}_{20} \mathrm{H}_{31} \mathrm{NO}_{4} \text { (349.4) } & \text { Ber. C } 68.76 \mathrm{H} 8.94 \text { N } 4.00 \\
& \text { Gef. C } 69.07 \text { H } 9.13 \text { N } 3.95
\end{array}
$$

Allgemeine Arbeitsvorschrift für die Darstellung der Aminosäuren 8a, b: Eine Lösung von $1 \mathrm{mmol} 7$ in ca. $10 \mathrm{ml} 6 \mathrm{~N} \mathrm{HCl}$ wird in einem Druckrohr $4 \mathrm{~h}$ auf $160^{\circ} \mathrm{C}$ (Badtemp.) erhitzt. Das Reaktionsgemisch wird anschließend $3 \mathrm{mal}$ mit $5 \mathrm{ml} \mathrm{CH}_{2} \mathrm{Cl}_{2}$ ausgeschüttelt, i.Vak. eingeengt, in wenig Wasser gelöst und die Lösung auf einen sauren Ionenaustauscher Dowex 50W-X8 gegeben. Die Säule wird so lange mit Wasser gewaschen, bis das abtropfende Wasser neutral ist und dann mit 10proz. $\mathrm{NH}_{4} \mathrm{OH}$-Lösung eluiert. Die Ninhydrinpositiven Fraktionen werden vereinigt und unter Erwärmen im Wasserstrahlvakuum eingeengt. Zur vollständigen Entfernung des Ammoniaks wird der Rückstand noch $2 \mathrm{mal}$ in Wasser aufgenommen und wie oben wieder davon befreit.

(2R)-2-Benzyl-2-piperidincarbonsäure (8a): Darstellung aus $0.403 \mathrm{~g}(0.101 \mathrm{mmol}) 7 \mathrm{a}$ in $11 \mathrm{ml} 6 \mathrm{~N} \mathrm{HCl}$, Extraktion mit $3 \mathrm{mal}$ $50 \mathrm{ml} \mathrm{Et}{ }_{2} \mathrm{O}$; Ausb. $0.176 \mathrm{~g}(80 \%)$ farblose Kristalle, Schmp. $350^{\circ} \mathrm{C}$ (Zers.), $[\alpha]_{\mathrm{D}}^{23}=-3.0(c=0.2$ in Wasser $) .-\mathrm{IR}(\mathrm{KBr}): \dot{v}=3103$ $\mathrm{cm}^{-1}, 2329,1616 .{ }^{-1} \mathrm{H}-\mathrm{NMR}\left(\mathrm{CD}_{3} \mathrm{OD}, 300 \mathrm{MHz}\right), \delta=1.23-1.34$ $(\mathrm{m}, 1 \mathrm{H}), 1.43-1.53(\mathrm{~m}, 2 \mathrm{H}), 1.54-1.75(\mathrm{~m}, 2 \mathrm{H}), 2.16(\mathrm{~d}$, breit, $J=12.9 \mathrm{~Hz}, 1 \mathrm{H}), 2.63\left(\mathrm{dt}, J=4.0 / 13 \mathrm{~Hz}, 1 \mathrm{H}, \mathrm{NHCH}_{\mathrm{ax}}\right), 2.69$ (d, $J=13.1 \mathrm{~Hz}, 1 \mathrm{H}, H \mathrm{CHPh}), 2.78$ (d, breit, $J=13 \mathrm{~Hz}, 1 \mathrm{H}$, $\left.\mathrm{NHC}_{\text {eq }}\right), 2.91(\mathrm{~d}, J=13.1 \mathrm{~Hz}, 1 \mathrm{H}, \mathrm{HCHPh}), 7.17-7.20(\mathrm{~d}, J=$ $6.7 \mathrm{~Hz}, 2 \mathrm{H}$, arom.), 7.29-7.34 (m, 3H, arom.). - MS (70 eV): $m / z=219\left[\mathrm{M}^{+}\right], 174\left[\mathrm{M}^{+}-45\right], 128\left[\mathrm{M}^{+}-91\right]$.

$$
\begin{array}{ll}
\mathrm{C}_{13} \mathrm{H}_{17} \mathrm{NO}_{2} \text { (219.2) } & \text { Ber. C } 71.23 \mathrm{H} 7.81 \text { N } 6.38 \\
& \text { Gef. C } 71.41 \text { H } 7.90 \text { N } 6.08
\end{array}
$$

(2S)-2-Methyl-2-piperidincarbonsäure (8b): Dargestellt aus $1.045 \mathrm{~g}(3.230 \mathrm{mmol}) 7 \mathrm{~b}$ in $30 \mathrm{ml} 6 \mathrm{~N} \mathrm{HCl}$; Ausb. $0.350 \mathrm{~g}(76 \%)$ farblose Kristalle, Schmp. $360^{\circ} \mathrm{C}$ (Zers.). [Lit. ${ }^{[3]} 360^{\circ} \mathrm{C}$ (Zers.)], $[\alpha]_{\mathrm{D}}^{23}=-3.7(c=0.2$ in Wasser $)\left\{\right.$ Lit. $^{[7]}[\alpha]_{\mathrm{D}}^{23}=-3.7(c=0.2$ in Wasser)\}.

(2R)-2-Allyl-2-piperidincarbonsäure (8c): $0.180 \mathrm{~g}$ (0.515 mmol) 7c wurden mit $0.371 \mathrm{~g}(15.47 \mathrm{mmol}) \mathrm{LiOH}$ in $6 \mathrm{ml}$ Ethylenglycolmonomethylether $48 \mathrm{~h}$ in einem Druckrohr auf $160^{\circ} \mathrm{C}$ (Badtemp.) erhitzt. Anschließend wurde i.Vak. vom Lösungsmittel befreit, in $10 \mathrm{ml}$ Wasser gelöst, mit $0.079 \mathrm{~g} \mathrm{Ammoniumchlorid} \mathrm{versetzt} \mathrm{und}$ auf ca. $3 \mathrm{ml}$ (i.Vak.) eingeengt. Das Produkt wurde analog zu 7a durch Ionenaustauschchromatographie isoliert; Ausb. $0.056 \mathrm{~g}$ $(64 \%)$ farblose Kristalle, Schmp. $280^{\circ} \mathrm{C}$ (Zers.), $[\alpha]_{\mathrm{D}}^{25}=+2.8(c=$ 0.8 in Wasser). - IR (KBr): $\tilde{v}=3425 \mathrm{~cm}^{-1}, 3068,2317,1610$. ${ }^{1} \mathrm{H}-\mathrm{NMR}\left(\mathrm{D}_{2} \mathrm{O}, 300 \mathrm{MHz}\right): \delta=1.34-1.37(\mathrm{~m}, 1 \mathrm{H}), 1.41-1.52(\mathrm{~m}$, $2 \mathrm{H}), 1.57-1.73(\mathrm{~m}, 2 \mathrm{H}), 2.20(\mathrm{~d}$, breit, $J=13.9 \mathrm{~Hz}, 1 \mathrm{H}), 2.37$ (dd, $\left.J=8.6 / 14 \mathrm{~Hz}, 1 \mathrm{H}, \mathrm{HCHCH}=\mathrm{CH}_{2}\right), 2.53(\mathrm{dd}, J=6.5 / 14 \mathrm{~Hz}, 1 \mathrm{H}$, $\left.\mathrm{HCHCH}=\mathrm{CH}_{2}\right), \quad 2.99-3.31 \quad(\mathrm{~m}, 2 \mathrm{H}), \quad 5.03-5.23 \quad(\mathrm{~m}, 2 \mathrm{H}$, $\mathrm{CH}=\mathrm{CH}), 5.63-5.80\left(\mathrm{~m}, 1 \mathrm{H}, \mathrm{CH}=\mathrm{CH}_{2}\right) .-\mathrm{MS}(70 \mathrm{eV}): \mathrm{m} / \mathrm{z}=$ $169\left[\mathrm{M}^{+}\right], 128\left[\mathrm{M}^{+}-41\right], 124\left[\mathrm{M}^{+}-45\right], 89\left[\mathrm{M}^{+}-86\right]$.

$$
\mathrm{C}_{9} \mathrm{H}_{15} \mathrm{NO}_{2} \text { (169.2) } \quad \text { Ber. C } 63.88 \text { H } 8.93 \text { N } 8.27
$$

(2S)-Methyl-2-[(IS,3R)-1-benzyloxy-3-methoxymethyl-2,2,3-trimethylcyclopentylcarbonylamino]-3-phenylpropionat (9a): $0.176 \mathrm{~g}$ $(0.580 \mathrm{mmol}) 1$ wurden in $1.5 \mathrm{ml} \mathrm{CH}_{2} \mathrm{Cl}_{2}$ bei $0^{\circ} \mathrm{C}$ mit $0.148(1.172$ mmol, $0.09 \mathrm{ml}$ ) Oxalylchlorid und einem Tropfen DMF versetzt. Das Gemisch wurde 5 min bei $0^{\circ} \mathrm{C}$ und danach bis zur Beendigung der Gasentwicklung (ca. 2 h) bei Raumtemp. gerührt. Dann wurde i.Vak. eingeengt, 30 min Hochvak. angelegt, der erhaltene Rückstand in $1.5 \mathrm{ml}$ THF gelöst und mit $0.253 \mathrm{~g}$ (1.173 mmol) $(S)$-Phenylalaninmethylester in $2 \mathrm{ml}$ THF versetzt. Nach einstündigem Rühren bei Raumtemp. wurde THF i.Vak. verdampft und die Probe in $30 \mathrm{ml} \mathrm{Et}_{2} \mathrm{O}$ aufgenommen. Die Etherphase wurde zunächst mit $0.1 \mathrm{~N}$ Salzsäure und dann mit $0.1 \mathrm{~N} \mathrm{NaOH}$ gewaschen und die alkalische Phase mit $\mathrm{Et}_{2} \mathrm{O}$ rückextrahiert $(3 \times 30 \mathrm{ml})$. Die Etherphasen wurden vereinigt, getrocknet $\left(\mathrm{MgSO}_{4}\right)$ und i.Vak. eingeengt. Der Rückstand wurde durch SC ( $n$-Hexan/EtOAc, 6:4) gereinigt; Ausb. $0.237 \mathrm{~g}(87 \%)$ farbloses Öl, $[\alpha]_{546}^{20}=+25.8,[\alpha]_{578}^{20}=$ $+22.2\left(c=0.7\right.$ in Diethylether). - IR (Film): $\tilde{v}=3080 \mathrm{~cm}^{-1}, 1750$, 1680. $-{ }^{1} \mathrm{H}-\mathrm{NMR}\left(\mathrm{CDCl}_{3}, 400 \mathrm{MHz}\right): \delta=0.53\left(\mathrm{~s}, 3 \mathrm{H}, \mathrm{CH}_{3}\right), 0.65$ (s, $\left.3 \mathrm{H}, \mathrm{CH}_{3}\right), 0.91$ (s, $\left.3 \mathrm{H}, \mathrm{CH}_{3}\right), 1.42-1.46$ (m, $\left.1 \mathrm{H}, \mathrm{cPent}\right)$, $1.82-1.88$ (m, 1 H, cPent), 1.96-2.04 (m, 1H, cPent), 2.50-2.56 (m, $1 \mathrm{H}, \mathrm{cPent}), 2.89$ (dd, $J=8.6 / 14 \mathrm{~Hz}, 1 \mathrm{H}, H \mathrm{CHPh}), 3.08$ (d, $\left.J=8.5 \mathrm{~Hz}, 1 \mathrm{H}, \mathrm{HCHOCH}{ }_{3}\right), 3.16(\mathrm{dd}, J=5.1 / 14 \mathrm{~Hz}, 1 \mathrm{H}$, $\mathrm{HCHPh}), 3.25\left(\mathrm{~s}, 3 \mathrm{H}, \mathrm{OCH}_{3}\right), 3.50(\mathrm{~d}, J=8.5 \mathrm{~Hz}, 1 \mathrm{H}$, $\left.H \mathrm{CHOCH}_{3}\right), 3.68\left(\mathrm{~s}, 3 \mathrm{H}, \mathrm{OCH}_{3}\right), 4.26(\mathrm{~d}, J=11.1 \mathrm{~Hz}, 1 \mathrm{H}$, $\left.\mathrm{OCH}_{2} \mathrm{Ph}\right), 4.45\left(\mathrm{~d}, J=11.1 \mathrm{~Hz}, 1 \mathrm{H}, \mathrm{OCH}_{2} \mathrm{Ph}\right), 4.82-4.87(\mathrm{~m}, 1 \mathrm{H}$, $\left.\mathrm{NCHCH}_{2} \mathrm{Ph}\right), 6.64$ (d, breit, $\left.J=7.7 \mathrm{~Hz}, 1 \mathrm{H}, \mathrm{NH}\right), 7.06(\mathrm{~d}, J=$ $6.4 \mathrm{~Hz}, 2 \mathrm{H}$, arom.), $7.20-7.36(\mathrm{~m}, 8 \mathrm{H}$, arom.). - MS $(70 \mathrm{eV})$ : $m / z=467\left[\mathrm{M}^{+}\right]$.

$$
\begin{array}{ll}
\mathrm{C}_{28} \mathrm{H}_{37} \mathrm{NO}_{5} \text { (467.6) } & \text { Ber. C 71.92 H } 7.92 \text { N } 2.99 \\
& \text { Gef. C 72.21 H } 8.16 \text { N } 2.88
\end{array}
$$

(2S)-Ethyl-2-[(1S,3R)-1-benzyloxy-3-methoxymethyl-2,2,3-trimethylcyclopentylcarbonylamino ]-3-phenylpropionat (9b): Die Darstellung erfolgte analog zur Synthese von 9a aus $0.220 \mathrm{~g}(0.718$ mmol) 1 und $0.240 \mathrm{~g} \mathrm{(1.25} \mathrm{mmol)} \mathrm{(S)-Phenylalaninethylester;} \mathrm{Ausb.}$ $0.328 \mathrm{~g}(93 \%)$ farbloses Öl, $[\alpha]_{546}=+30.2,[\alpha]_{578}=+26.9(c=0.5$ in Diethylether). - IR (Film): $\tilde{v}=3354 \mathrm{~cm}^{-1}, 3030,1738,1675$. - ${ }^{1} \mathrm{H}-\mathrm{NMR}\left(\mathrm{CDCl}_{3}, 400 \mathrm{MHz}\right): \delta=0.54\left(\mathrm{~s}, 3 \mathrm{H}, \mathrm{CH}_{3}\right), 0.65$ (s, $\left.3 \mathrm{H}, \mathrm{CH}_{3}\right), 0.90\left(\mathrm{~s}, 3 \mathrm{H}, \mathrm{CH}_{3}\right), 1.20\left(\mathrm{t}, J=7.3 \mathrm{~Hz}, 3 \mathrm{H}, \mathrm{OCH}_{2} \mathrm{CH}_{3}\right)$, $1.43-1.50$ (m, 1 H, cPent), 1.80-1.87 (m, 1 H, cPent), 1.96-2.03 (m, 1H, cPent), 2.51-2.59 (m, 1H, cPent), 2.92 (dd, $J=8.6 /$ $\left.14.1 \mathrm{~Hz}, 1 \mathrm{H}, \mathrm{NCCH}_{2} \mathrm{Ph}\right), 3.08\left(\mathrm{~d}, J=8.6 \mathrm{~Hz}, 1 \mathrm{H}, \mathrm{HCHOCH}_{3}\right)$, $3.16\left(\mathrm{dd}, J=5.6 / 14.1 \mathrm{~Hz}, 1 \mathrm{H}, \mathrm{NCCH}_{2} \mathrm{Ph}\right), 3.25\left(\mathrm{~s}, 3 \mathrm{H}, \mathrm{OCH}_{3}\right)$, $3.50\left(\mathrm{~d}, J=8.6 \mathrm{~Hz}, 1 \mathrm{H}, H \mathrm{CHOCH}_{3}\right), 4.11-4.19(\mathrm{~m}, 2 \mathrm{H}$, $\left.\mathrm{OCH}_{2} \mathrm{CH}_{3}\right), 4.19\left(\mathrm{~d}, J=11 \mathrm{~Hz}, 1 \mathrm{H}, \mathrm{OCH}_{2} \mathrm{Ph}\right), 4.44(\mathrm{~d}, J=11 \mathrm{~Hz}$, $\left.1 \mathrm{H}, \mathrm{OCH}_{2} \mathrm{Ph}\right), 4.79-4.84\left(\mathrm{~m}, 1 \mathrm{H}, \mathrm{NCHCH}_{2} \mathrm{Ph}\right), 6.65$ (d, breit, $J=$ $12.1 \mathrm{~Hz}, 1 \mathrm{H}, \mathrm{NH}), 7.08(\mathrm{~d}, J=7.7 \mathrm{~Hz}, 2 \mathrm{H}$, arom. $), 7.18-7.37(\mathrm{~m}$, $8 \mathrm{H}$, arom. $)$ - $\mathrm{MS}(70 \mathrm{eV}): m / z=481\left[\mathrm{M}^{+}\right]$.

$$
\begin{array}{ll}
\mathrm{C}_{29} \mathrm{H}_{39} \mathrm{NO}_{5} \text { (481.6) } & \text { Ber. C } 72.32 \mathrm{H} 8.16 \text { N } 2.90 \\
& \text { Gef. C } 72.11 \mathrm{H} 8.20 \text { N } 2.96
\end{array}
$$

(5S,7R)-7-Methoxymethyl-6.6,7-trimethyl-1,3-dioxaspiro[4.4]nonan-2,4-dion $(\mathbf{1 0})$ : $0.285 \mathrm{~g}(1.321 \mathrm{mmol}) \mathbf{1}^{[5]}$ wurden in $2 \mathrm{ml}$ THF gelöst und nach Zugabe von $1.800 \mathrm{mmol}(0.217 \mathrm{ml})$ Chlorameisensäure-trichlormethylester $4 \mathrm{~h}$ unter Rückfluß erhitzt. Nach Entfernung des Lösungsmittels i.Vak. wurde aus $n$-Hexan umkristallisiert; Ausb. $0.180 \mathrm{~g}(56 \%)$ farblose Kristalle, Schmp. $83^{\circ} \mathrm{C}$, $[\alpha]_{546}=-42.6,[\alpha]_{578}=-33.3\left(c=0.8\right.$ in $\left.\mathrm{Et}_{2} \mathrm{O}\right) .-\operatorname{IR}(\mathrm{KBr}): \tilde{v}=$ $2968 \mathrm{~cm}^{-1}, 1703,1630 .-{ }^{1} \mathrm{H}-\mathrm{NMR}\left(\mathrm{CDCl}_{3}, 400 \mathrm{MHz}\right): \delta=1.01$ (s, $\left.3 \mathrm{H}, \mathrm{CH}_{3}\right), 1.08\left(\mathrm{~s}, 3 \mathrm{H}, \mathrm{CH}_{3}\right), 1.09\left(\mathrm{~s}, 3 \mathrm{H}, \mathrm{CH}_{3}\right), 1.55-1.60(\mathrm{~m}$, $1 \mathrm{H}, \mathrm{cPent}), 2.11-2.17$ (m, 1H, cPent), 2.21-2.22 (m, 1 H, cPent), $2.47-2.51$ (m, $1 \mathrm{H}, \mathrm{cPent}), 3.19$ (d, $J=8.8 \mathrm{~Hz}, 1 \mathrm{H}, \mathrm{CH}_{2} \mathrm{OCH}_{3}$ ), 3.33 (d, $J=8.8 \mathrm{~Hz}, 1 \mathrm{H}, \mathrm{CH}_{2} \mathrm{OCH}_{3}$ ), $3.33\left(\mathrm{~s}, 3 \mathrm{H}, \mathrm{OCH}_{3}\right.$ ). - MS $(70 \mathrm{eV}): m / z=242\left[\mathrm{M}^{+}\right]$. 


\section{$\mathrm{C}_{12} \mathrm{H}_{18} \mathrm{O}_{5}$ (242.2) Ber. C $59.56 \mathrm{H} 7.49$ Gef. C $59.28 \mathrm{H} 7.68$}

(2S)-Methyl-2-[(IS,3R)-1-hydroxy-3-methoxymethyl-2,2,3-trimethylcyclopentylcarbonylamino]-3-phenylpropionat (11a): A) 0.237 $\mathrm{g}(0.507 \mathrm{mmol}) 9 \mathrm{a}$ wurden in $30 \mathrm{ml} \mathrm{EtOH}$ gelöst, mit $0.034 \mathrm{~g} \mathrm{Pd} /$ $\mathrm{C}(10 \% \mathrm{Pd})$ versetzt und ca. $12 \mathrm{~h}$ bei Normaldruck hydriert. Nach Abfiltrieren des Katalysators und Einengen des Lösungsmittels wurde das Rohprodukt durch SC ( $n$-Hexan/EtOAc, 6:4) gereinigt; Ausb. $0.183 \mathrm{~g}(95 \%)$ farblose Kristalle, Schmp. $94^{\circ} \mathrm{C},[\alpha]_{546}=$ $-24.5,[\alpha]_{578}=-20.9(c=1.1$ in Diethylether $)$. $-\operatorname{IR}(\mathrm{KBr}): \tilde{v}=$ $3435 \mathrm{~cm}^{-1}, 3316,1741,1678 .{ }^{\prime} \mathrm{H}-\mathrm{NMR}\left(\mathrm{CDCl}_{3}, 400 \mathrm{MHz}\right): \delta=$ $0.62\left(\mathrm{~s}, 3 \mathrm{H}, \mathrm{CH}_{3}\right), 0.67$ (s, 3H, $\left.\mathrm{CH}_{3}\right), 0.80\left(\mathrm{~s}, 3 \mathrm{H}, \mathrm{CH}_{3}\right), 1.54-1.62$ (m, 2H, cPent), 1.87-1.95 (m, 1 H, cPent), 2.61-2.70 (m, 1H, cPent), 3.00 (dd, $J=8.1 / 14 \mathrm{~Hz}, 1 \mathrm{H}, \mathrm{HCHPh}$ ), 2.99 (d, $J=9.3 \mathrm{~Hz}$, $\left.1 \mathrm{H}, \mathrm{CH}_{2} \mathrm{OCH}_{3}\right), 3.19(\mathrm{dd}, J=5.6 / 14 \mathrm{~Hz}, 1 \mathrm{H}, H \mathrm{CHPh}), 3.23(\mathrm{~d}$, $\left.J=9.3 \mathrm{~Hz}, 1 \mathrm{H}, \mathrm{CH}_{2} \mathrm{OCH}_{3}\right), 3.37\left(\mathrm{~s}, 3 \mathrm{H}, \mathrm{OCH}_{3}\right), 3.72(\mathrm{~s}, 3 \mathrm{H}$, $\left.\mathrm{OCH}_{3}\right), 4.91-4.95(\mathrm{~m}, 1 \mathrm{H}, \mathrm{NCH}), 5.91(\mathrm{~s}, 1 \mathrm{H}, \mathrm{OH}), 7.17-7.28$ (m, $5 \mathrm{H}$, arom.), $\mathrm{NH}$ nicht lokalisierbar. - MS $(70 \mathrm{eV}): \mathrm{m} / \mathrm{z}=$ $377\left[\mathrm{M}^{+}\right]$.

$$
\begin{array}{ll}
\mathrm{C}_{21} \mathrm{H}_{31} \mathrm{NO}_{5} \text { (377.5) } & \text { Ber. C } 66.81 \mathrm{H} 8.27 \text { N } 3.71 \\
& \text { Gef. C } 66.60 \mathrm{H} 8.28 \text { N } 3.59
\end{array}
$$

B) Eine Mischung aus $0.624 \mathrm{~g}(2.888 \mathrm{mmol}) 12$ und $0.491 \mathrm{~g} \mathrm{(3.028}$ mmol) $1,1^{\prime}$-Carbonyldiimidazol (CDI) in $30 \mathrm{ml}$ THF wurde ca. $12 \mathrm{~h}$ bei Raumtemp. gerührt und nach Zusatz von $1.146 \mathrm{~g}$ (5.931 mmol) (S)-Phenylalaninethylester (in $40 \mathrm{ml}$ THF) $8 \mathrm{~h}$ unter Rück$\mathrm{flu} B$ erhitzt. Dann wurde i.Vak. eingeengt, mit $100 \mathrm{ml} \mathrm{Et}_{2} \mathrm{O}$ und $20 \mathrm{ml} \mathrm{H}_{2} \mathrm{O}$ versetzt und mit $0.1 \mathrm{~N} \mathrm{HCl}$ angesäuert. Die wäßrige Phase wurde noch zweimal mit je $50 \mathrm{ml} \mathrm{Et}_{2} \mathrm{O}$ extrahiert. Die vereinigten organischen Extrakte wurden dann dreimal mit $0.05 \mathrm{~N}$ $\mathrm{NaOH}$ gewaschen, getrocknet $\left(\mathrm{MgSO}_{4}\right)$ und i.Vak. eingeengt. Der Rückstand wurde durch Zentrifugalchromatographie gereinigt; Ausb. $0.955 \mathrm{~g}(85 \%)$.

(2S)-Ethyl-2-[(1S,3R)-1-hydroxy-3-methoxymethyl-2,2,3-trimethylcyclopentylcarbonylamino ]-3-phenylpropionat (11b): A) $0.469 \mathrm{~g}$ $(0.47 \mathrm{mmol}) 9$ wurden in $30 \mathrm{ml} \mathrm{EtOH}$ gelöst, mit $0.034 \mathrm{~g} \mathrm{Pd} / \mathrm{C}(10 \%$ Pd) versetzt und unter Schütteln ca. $12 \mathrm{~h}$ bei Normaldruck hydriert. Dann wurde filtriert, i.Vak. eingeengt und durch SC $(n-\mathrm{He}-$ xan/EtOAc, 6:4) gereinigt; Ausb. $0.179 \mathrm{~g}(97 \%)$ farblose Kristalle, Schmp. $97^{\circ} \mathrm{C},[\alpha]_{546}=-20.1,[\alpha]_{578}=-18.4(c=0.99$ in Diethylether). IR $(\mathrm{KBr}): \tilde{v}=3392 \mathrm{~cm}^{-1}, 3086,2974,1741,1678 .{ }^{\prime} \mathrm{H}-$ NMR $\left(\mathrm{CDCl}_{3}, 400 \mathrm{MHz}\right): \delta=0.64\left(\mathrm{~s}, 3 \mathrm{H}, \mathrm{CH}_{3}\right), 0.69(\mathrm{~s}, 3 \mathrm{H}$, $\left.\mathrm{CH}_{3}\right), 0.80\left(\mathrm{~s}, 3 \mathrm{H}, \mathrm{CH}_{3}\right), 0.82-0.89(\mathrm{~m}, 1 \mathrm{H}, \mathrm{cPent}), 1.22(\mathrm{t}, J=$ $\left.7.3 \mathrm{~Hz}, 3 \mathrm{H}, \mathrm{OCH}_{2} \mathrm{CH}_{3}\right), 1.58-1.62(\mathrm{~m}, 1 \mathrm{H}$, cPent), $1.87-1.95$ (m, $1 \mathrm{H}$, cPent), 2.62-2.70 (m, 1H, cPent), 2.98-3.04 (m, 2H, $\mathrm{CHOCH}_{3}$ und $H \mathrm{CHPh}$ ), 3.18 (dd, $J=5.6 / 14.1 \mathrm{~Hz}, 1 \mathrm{H}, \mathrm{CH}_{2} \mathrm{Ph}$ ), $3.23\left(\mathrm{~d}, J=9.4 \mathrm{~Hz}, 1 \mathrm{H}, \mathrm{CH}_{2} \mathrm{OCH}_{3}\right), 3.37\left(\mathrm{~s}, 3 \mathrm{H}, \mathrm{OCH}_{3}\right), 4.18(\mathrm{q}$, $\left.J=7.3 \mathrm{~Hz}, 2 \mathrm{H}, \mathrm{OCH}_{2} \mathrm{CH}_{3}\right), 4.85-4.91\left(\mathrm{~m}, 1 \mathrm{H}, \mathrm{CHCOOC}_{2} \mathrm{H}_{5}\right)$, $5.88(\mathrm{~s}, 1 \mathrm{H}, \mathrm{OH}), 7.20-7.30(\mathrm{~m}, 5 \mathrm{H}$, arom.), NH nicht lokalisierbar. - MS $(70 \mathrm{eV}): m / z=391\left[\mathrm{M}^{+}\right]$.

$$
\begin{array}{lll}
\mathrm{C}_{22} \mathrm{H}_{33} \mathrm{NO}_{5} \text { (391.5) } & \text { Ber. C } 67.49 \mathrm{H} 8.49 \text { N } 3.58 \\
& \text { Gef. C } 67.55 \text { H } 8.64 \text { N } 3.45
\end{array}
$$

B) Eine Mischung aus $0.143 \mathrm{~g}(0.590 \mathrm{mmol}) \mathbf{1 0}$ und $0.203 \mathrm{~g}(0.866$ mmol) (S)-Phenylalanin-ethylester in $4 \mathrm{ml}$ THF wurde $12 \mathrm{~h}$ bei Raumtemp. gerührt. Dann wurde i.Vak. eingeengt, in $30 \mathrm{ml} \mathrm{Et}_{2} \mathrm{O}$ aufgenommen und mit $20 \mathrm{ml} \mathrm{H}_{2} \mathrm{O}$ und $5 \mathrm{ml} 0.1 \mathrm{~N} \mathrm{HCl}$ versetzt. Die wäßrige Phase wurde noch mit $\mathrm{Et}_{2} \mathrm{O}$ extrahiert, und die vereinigten organischen Phasen wurden mit Wasser $(3 \times 20 \mathrm{ml})$ und $0.05 \mathrm{~N}$ $\mathrm{NaOH}(1 \times 5 \mathrm{ml})$ gewaschen, getrocknet $\left(\mathrm{MgSO}_{4}\right)$ und i.Vak. eingeengt. Der Rückstand wurde durch SC ( $n$-Hexan/EtOAc, 6:4), gereinigt, Ausb. $0.114 \mathrm{~g}(59 \%)$.

C) Eine Mischung aus $0.394 \mathrm{~g}(1.824 \mathrm{mmol}) 12$ und $0.311 \mathrm{~g}(1.919$ mmol) CDI in $19 \mathrm{ml}$ THF wurde ca. $12 \mathrm{~h}$ bei Raumtemp. gerührt und nach Zusatz von $0.526 \mathrm{~g}(2.725 \mathrm{mmol})(S)$-Phenylalaninethylester $8 \mathrm{~h}$ unter Rückfluß erhitzt. Dann wurde i.Vak. eingeengt, mit $50 \mathrm{ml} \mathrm{Et}{ }_{2} \mathrm{O}$ und $15 \mathrm{ml} \mathrm{H} \mathrm{H}_{2} \mathrm{O}$ versetzt und mit $0.1 \mathrm{~N} \mathrm{HCL}$ angesäuert.

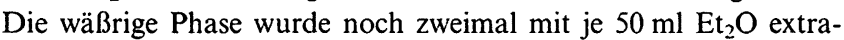
hiert. Die vereinigten organischen Extrakte wurden dann dreimal mit $0.05 \mathrm{~N} \mathrm{NaOH}$ gewaschen, getrocknet $\left(\mathrm{MgSO}_{4}\right)$ und i.Vak. eingeengt. Der Rückstand wurde durch Zentrifugalchromatographie gereinigt; Ausb. $0.596 \mathrm{~g} \mathrm{(84 \% ).}$

(2S)-2-[(IS,3R)-1-Hydroxy-3-methoxymethyl-2,2,3-trimethylcyclopentylcarbonylamino]-3-phenylpropionsäure (13): A) $0.184 \mathrm{~g}$ $(0.487 \mathrm{mmol}) 11 \mathrm{a}$ und $0.120 \mathrm{~g} \mathrm{LiOH}$ wurden in einer Mischung aus $3 \mathrm{ml} \mathrm{CH} \mathrm{CH}_{3} \mathrm{OH}$ und $3 \mathrm{ml} \mathrm{H}_{2} \mathrm{O} 1 \mathrm{~h}$ unter Rückfluß erhitzt. Dann wurde i.Vak. auf ca. $3 \mathrm{ml}$ eingeengt, mit $20 \mathrm{ml} \mathrm{H}_{2} \mathrm{O}$ versetzt und mit $0.2 \mathrm{~N} \mathrm{HCl}$ auf $\mathrm{pH}=2-3$ eingestellt. Die wäßrige Phase wurde mit $\mathrm{Et}_{2} \mathrm{O}(3 \times 50 \mathrm{ml})$ ausgeschüttelt, die Extrakte wurden vereinigt, getrocknet $\left(\mathrm{MgSO}_{4}\right)$ und i.Vak. vom Lösungsmittel befreit. Das Produkt wurde durch Zentrifugalchromatographie (MeOH/EtOAc, 4:6) gereinigt; Ausb. $0.130 \mathrm{~g}(78 \%)$ farblose Kristalle, Schmp. $162^{\circ} \mathrm{C},[\alpha]_{546}=-2.4,[\alpha]_{578}=-2.3,(c=0.25$ in Ethanol $)$. IR $(\mathrm{KBr}): \tilde{v}=3405 \mathrm{~cm}^{-1}, 3062,2975,1650,1600 .-{ }^{1} \mathrm{H}-\mathrm{NMR}$ $\left(\mathrm{CD}_{3} \mathrm{OD}, 400 \mathrm{MHz}\right): \delta=0.48\left(\mathrm{~s}, 3 \mathrm{H}, \mathrm{CH}_{3}\right), 0.61\left(\mathrm{~s}, 3 \mathrm{H}, \mathrm{CH}_{3}\right)$, 0.82 (s, $\left.3 \mathrm{H}, \mathrm{CH}_{3}\right), 1.42-1.52$ (m, $2 \mathrm{H}$, cPent), 1.87-1.94 (m, $1 \mathrm{H}$, cPent), 2.53-2.62 (m, 1 H, cPent), 2.97 (dd, $J=9.0 / 14.1 \mathrm{~Hz}, 1 \mathrm{H}$, $\left.\mathrm{CH}_{2} \mathrm{Ph}\right), 3.07\left(\mathrm{~d}, \mathrm{~J}=9 \mathrm{~Hz}, 1 \mathrm{H}, \mathrm{CHOCH}_{3}\right), 3.23-3.26(1 \mathrm{H}$, $\mathrm{CH}_{2} \mathrm{Ph}$, Signal zum Teil verdeckt), $3.32\left(\mathrm{~s}, 3 \mathrm{H}, \mathrm{OCH}_{3}\right), 3.44$ (d, $\left.J=9 \mathrm{~Hz}, 1 \mathrm{H}, \mathrm{CH}_{2} \mathrm{OCH}_{3}\right), 4.58\left(\mathrm{~m}, 1 \mathrm{H}, \mathrm{CHCH}_{2} \mathrm{Ph}\right), 7.12-7.26$ (m, 5H, arom.). - MS $(70 \mathrm{eV}): m / z=363\left[\mathrm{M}^{+}\right]$.

$$
\begin{array}{ll}
\mathrm{C}_{20} \mathrm{H}_{29} \mathrm{NO}_{5} \text { (363.4) } & \text { Ber. C } 66.10 \mathrm{H} 8.04 \text { N } 3.85 \\
& \text { Gef. C } 65.89 \mathrm{H} 8.21 \text { N } 3.65
\end{array}
$$

B) Aus $0.321 \mathrm{~g}(0.891 \mathrm{mmol}) \mathbf{1 1 b}$ in $5 \mathrm{ml} \mathrm{EtOH}$ und $5 \mathrm{ml} \mathrm{H}_{2} \mathrm{O}$ und $0.165 \mathrm{~g} \mathrm{NaOH}$ analog zur Darstellung aus 11a; Ausb. $0.285 \mathrm{~g}$ $(94 \%)$.

(2R,5S,8S)-8-Benzyl-2-methoxymethyl-1,1,2-trimethyl-6-oxa-9-azaspiro[4.5]decan-7,10-dion (14): $0.055 \mathrm{~g}(0.151 \mathrm{mmol}) 13$ und

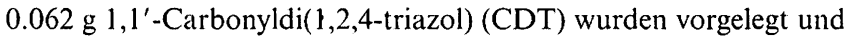
mit $1.5 \mathrm{ml} \mathrm{THF}$ versetzt. Es wurde erst $24 \mathrm{~h}$ bei Raumtemp. gerührt und anschließend für weitere $24 \mathrm{~h}$ auf $45^{\circ} \mathrm{C}$ erwärmt. Nach Zusatz von $2 \mathrm{ml} \mathrm{H}_{2} \mathrm{O}$ wurde i.Vak. eingeengt, der Rückstand in $5 \mathrm{ml} \mathrm{Was-}$ ser aufgenommen und die Lösung mit $0.1 \mathrm{~N} \mathrm{HCl}$ auf $\mathrm{pH}=2$ eingestellt. Die wäßrige Phase wurde dann mit $\mathrm{Et}_{2} \mathrm{O}(3 \times 30 \mathrm{ml})$ ausgeschüttelt. Die vereinigten organischen Extrakte wurden mit $0.1 \mathrm{~N}$ $\mathrm{NaOH}(3 \times 10 \mathrm{ml})$ gewaschen, getrocknet $\left(\mathrm{MgSO}_{4}\right)$ und i.Vak. vom Lösungsmittel befreit. Der Rückstand wurde durch SC ( $n$-Hexan/ EtOAc, 7:3) gereinigt; Ausb. $0.037 \mathrm{~g}(72 \%)$ farblose Kristalle, Schmp. $158^{\circ} \mathrm{C},[\alpha]_{546}^{20}=+2.8[\alpha]_{578}^{20}=+2.4(c=0.5$ in Hexan $)$. IR $(\mathrm{KBr}): \tilde{v}=3030 \mathrm{~cm}^{-1}, 2980,1770,1650 .-{ }^{1} \mathrm{H}-\mathrm{NMR}\left(\mathrm{CDCl}_{3}\right.$, $400 \mathrm{MHz}): \delta=0.94\left(\mathrm{~s}, 3 \mathrm{H}, \mathrm{CH}_{3}, 1.01\left(\mathrm{~s}, 3 \mathrm{H}, \mathrm{CH}_{3}\right), 1.04(\mathrm{~s}, 3 \mathrm{H}\right.$, $\mathrm{CH}_{3}$ ), 1.45-1.50 (m, 1H, cPent), 1.91-2.00 (m, 2H, cPent), $2.71-2.79$ (m, $1 \mathrm{H}$, cPent), 2.85 (dd, $J=10.5 / 14 \mathrm{~Hz}, 1 \mathrm{H}, \mathrm{CH}_{2} \mathrm{Ph}$ ), $3.25\left(\mathrm{~d}, J=9 \mathrm{~Hz}, 1 \mathrm{H}, \mathrm{CH}_{2} \mathrm{OCH}_{3}\right), 3.33\left(\mathrm{~s}, 3 \mathrm{H}, \mathrm{OCH}_{3}\right), 3.39$ (d, $\left.J=9 \mathrm{~Hz}, 1 \mathrm{H}, \mathrm{CH}_{2} \mathrm{OCH}_{3}\right), 3.59$ (dd, $J=3.5 / 14 \mathrm{~Hz}, 1 \mathrm{H}, \mathrm{CH}_{2} \mathrm{Ph}$ ), $4.35-4.38\left(\mathrm{dd}, J=3.5 / 10.5 \mathrm{~Hz}, 1 \mathrm{H}, \mathrm{NCHCH}_{2} \mathrm{Ph}\right), 5.68(\mathrm{~s}, 1 \mathrm{H}$, $\mathrm{NH}), 7.21-7.40\left(\mathrm{~m}, 5 \mathrm{H}\right.$, arom.). MS (70 eV): $\mathrm{m} / z=345\left[\mathrm{M}^{+}\right]$.

$$
\begin{array}{ll}
\mathrm{C}_{20} \mathrm{H}_{27} \mathrm{NO}_{4} \text { (345.4) } & \text { Ber. C } 69.54 \mathrm{H} 7.87 \mathrm{~N} 4.05 \\
& \text { Gef. C } 69.67 \mathrm{H} 8.07 \text { N } 3.91
\end{array}
$$

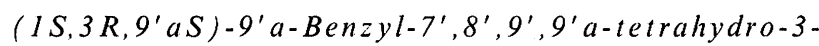
methoxymethyl-2,2,3-trimethylspiro [cyclopentan-1,3' (4' $H)$ pyrido[2,1-c][1,4]oxazin]-1' (6' H), $4^{\prime}$-dion (15): $\mathrm{Zu} 0.019 \mathrm{~g}(0.055$ mmol) 14 in $1 \mathrm{ml} \mathrm{THF}$ wurden bei $-78^{\circ} \mathrm{C} 0.078 \mathrm{ml}(0.11 \mathrm{mmol}) \mathrm{s}$ BuLi und nach $20 \mathrm{~min} 0.5 \mathrm{ml} \mathrm{3,4,5,6-Tetrahydro-1,3-dimethyl-}$ 2(1H)-pyrimidinon zugespritzt. Anschließend wurden $0.012 \mathrm{~g}$ (0.055 mmol) 1,4-Dibrombutan zugefügt. Nach zweistündigem 
Rühren bei $-78^{\circ} \mathrm{C}$ und einstündigem Rühren bei $0^{\circ} \mathrm{C}$ wurde mit $2 \mathrm{ml}$ Eis/Wasser versetzt. Dann wurde das Lösungsmittel iVak. entfernt, der Rückstand in $30 \mathrm{ml} \mathrm{Et}_{2} \mathrm{O}$ aufgenommen und $3 \mathrm{mal} \mathrm{mit}$ $10 \mathrm{ml} 0.05 \mathrm{~N} \mathrm{HCl}$ und $3 \mathrm{mal}$ mit $10 \mathrm{ml} 0.05 \mathrm{~N} \mathrm{NaOH}$ gewaschen. Die organische Phase wurde mit $\mathrm{MgSO}_{4}$ getrocknet und i. Vak. eingeengt. Der erhaltene Rückstand wurde durch SC (n-Hexan/ EtOAc, 7:3) gereinigt; Ausb. $0.007 \mathrm{~g} \mathrm{(32 \% )} \mathrm{farblose} \mathrm{Kristalle,}$ Schmp. $104^{\circ} \mathrm{C},[\alpha]_{546}^{20}=-5.0,[\alpha]_{578}^{20}=-9.1\left(c=0.35\right.$ in $\left.\mathrm{CH}_{2} \mathrm{Cl}_{2}\right)$. IR (KBr): $\tilde{v}=1728 \mathrm{~cm}^{-1}, 1657 .{ }^{1} \mathrm{H}-\mathrm{NMR}\left(\mathrm{CDCl}_{3}, 300 \mathrm{MHz}\right):$ $\delta=0.46$ (s, 3H, $\left.\mathrm{CH}_{3}\right), 0.88-0.93$ (m, 1 H, Pip.), 0.93 (s, 3H, $\left.\mathrm{CH}_{3}\right)$, 1.25 (s, 3H, $\left.\mathrm{CH}_{3}\right), 1.37-1.47$ (m, 1H, cPent), 1.78-1.90 (m, $6 \mathrm{H}$, Pip. und cPent), 2.22 (d, breit, $J=13.0 \mathrm{~Hz}, 1 \mathrm{H}), 2.51-2.53$ (m, $1 \mathrm{H}$, cPent), 2.99 (d, $\left.J=9.0 \mathrm{~Hz}, 1 \mathrm{H}, \mathrm{CH}_{2} \mathrm{OCH}_{3}\right), 3.10$ (dt, $J=3.0 /$ $\left.13 \mathrm{~Hz}, 1 \mathrm{H}, \mathrm{NHCH} \mathrm{ax}_{\mathrm{ax}}\right), 3.20\left(\mathrm{~d}, J=14.1 \mathrm{~Hz}, 1 \mathrm{H}, \mathrm{CH}_{2} \mathrm{Ph}\right), 3.23(\mathrm{~d}$, $\left.J=9.0 \mathrm{~Hz}, 1 \mathrm{H}, \mathrm{CH}_{2} \mathrm{OCH}_{3}\right), 3.25\left(\mathrm{~s}, 3 \mathrm{H}, \mathrm{OCH}_{3}\right), 3.62(\mathrm{~d}, J=$ $14.1 \mathrm{~Hz}, 1 \mathrm{H}, \mathrm{CH}_{2} \mathrm{Ph}$ ), 4.78 (d, breit, $J=13 \mathrm{~Hz}, 1 \mathrm{H}, \mathrm{NHCH}_{\mathrm{eq}}$ ), 7.14-7.16 (m, 2H, arom.), 7.24-7.26 (m, 3H, arom.). - MS $(70 \mathrm{eV}): m / z=399\left[\mathrm{M}^{+}\right]$.

$$
\mathrm{C}_{24} \mathrm{H}_{33} \mathrm{NO}_{4} \text { (399.5) Ber. C } 72.15 \mathrm{H} 8.32 \mathrm{~N} 3.50
$$
Gef. C 72.38 H 8.33 N 3.37

Allgemeine Arbeitsvorschrift für die Derivatisierung der Aminosäuren 8 mit $(R)$ - und (S)-1-(1-Naphthyl)ethylisocyanat $[(R)-16$ bzw. $(S)$-16] nach Lit. ${ }^{[8]}$ : $0.6 \mathrm{mmol}(R)-16$ bzw. $(S)-16$ werden in $9 \mathrm{ml}$ absol. Aceton gelöst und unter starkem Rühren unmittelbar darauf zu $0.02 \mathrm{mmol} 8$ in $3 \mathrm{ml} 4 \mathrm{M}$ Boratpuffer $(\mathrm{pH}=9)$ gegeben. Der sich dabei bildende, voluminöse Niederschlag wird nach 15 min abzentrifugiert und die überstehende Lösung 3 mal mit $5 \mathrm{ml}$ Cyclohexan/Diethylether [1/1, gesättigt mit Boratpuffer $(\mathrm{pH}=9)]$ ausgeschüttelt. Die wäßrige Phase wird mit $2 \mathrm{~N} \mathrm{HCl}$ angesäuert und $3 \mathrm{mal} \mathrm{mit} \mathrm{je} 10 \mathrm{ml}$ Diethylether extrahiert. Die vereinigten Etherphasen werden getrocknet $\left(\mathrm{MgSO}_{4}\right)$ und i.Vak. eingeengt. Der erhaltene Rückstand wird für die liquidchromatographische Stereoselektivitätsbestimmung herangezogen. - HPLC: Säule LiChrospher Si 60, $5 \mu, 25 \mathrm{~cm}$, Fließmittel: $n$-Hexan/Ethylacetat (9:1); Flußrate: $2 \mathrm{ml} / \mathrm{min}$; Detektion: UV, $254 \mathrm{~nm}$ Retentionszeiten [in min] für 17a: 22.9 , für 18 a 20.4 , für $17 \mathbf{b}: 39.10$, für $\mathbf{1 8 b}$ : 36.83 , für 17c: 16.94 ; für 18c: 14.50 . is Herrn Prof. Dr. H.-D. Stachel mit den besten Wünschen zum 65. Geburtstag gewidmet.

[1] Für Übersichtsartikel siehe: [1a] G. C. Barrett (Hrsg.), Chemistry and Biochemistry of the Amino Acids, Chapman and Hall, London, 1985. - ${ }^{[16]}$ I. Wagner, H. Musso, Angew. Chem. 1983, 95 , 827-839; Angew. Chem. Int. Ed. Engl. 1983, 22, 816-827. ${ }^{[1 \mathrm{c}]} \mathrm{R}$. M. Williams, Synthesis of Optically Active a-Amino Acids, Pergamon Press, Oxford, 1989. - ${ }^{[\mathrm{Id}]}$ M. J. O'Donnell, Tetrahedron 1988, 44, 5253.

[2] Siehe z. B.: [2a] U. Schöllkopf, U. Groth, C. Deng, Angew. Chem. 1981, 93, 793-795; Angew. Chem. Int. Ed. Engl. 1981, 20, 798-799. - [2b] U. Schöllkopf, R. Scheuer, Liebigs Ann. Chem. 1984, 939-950. - ${ }^{[2 c]}$ U. Groth, U. Schöllkopf, Y.-C. Chiang, Synthesis, 1982, 864-866. - [2d] M. Gander-Coquoz, D. Seebach, Helv. Chim. Acta 1988, 71, 224-236. - [2c] T. Weber, R. Aeschimann, T. Maetzke, D. Seebach, Helv. Chim. Acta 1986, 69, 1365-1377. - ${ }^{[2]}$ D. Seebach, E. Juaristi, D. D. Miller, C. Schickli, T. Weber, Helv. Chim. Acta 1987, 70, 237-270. ${ }^{[2 g]}$ D. Seebach, H. M. Bürger, C. P. Schickli, Liebigs Ann. Chem. 1991, 669-684. - [2h] A. Fadel, J. Salaün, Tetrahedron Lett., 1987, 28, 2243-2246. - ${ }^{[2 i]}$ S. Karady, J. S. Amato, L. M. Weinstock, Tetrahedron Lett. 1984, 25, 4337-4340. - ${ }^{[2 j]}$ R. M. Williams, M.-N. Im, J. Am. Chem. Soc., 1991, 113, 9276-9286.

[3] [3a] U. Schöllkopf, R. Hinrichs, R. Lonsky, Angew. Chem. 1987, 99, 137-138.; Angew. Chem. Int. Ed. Engl. 1987, 26, 143-145. - [3b] U. Schöllkopf, R. Wick, R. Hinrichs, M. Lange, Liebigs Ann. Chem. 1988, 1025-1031. - ${ }^{[3 c]}$ D. Seebach, M. Boes, R. Naef, W. B. Schweizer, J. Am. Chem. Soc. 1983, 105, 5390-5398. - ${ }^{[3 d]}$ D. Seebach, T. Weber, Helv. Chim. Acta, 1984, 67, 1650-1661. - ${ }^{[3 e]}$ D. Seebach, T. Weber, Helv. Chim. Acta 1985, 68, 155-161. - ${ }^{[3]}$ D. Seebach, E. Dziadulewicz, L. Behrendt, S. Cantoreggi, R. Fitzi, Liebigs Ann. Chem. 1989, 1215-1232. - [3g] G. I. Georg, X. Guan, J. Kant, Biorg. Med. Chem. Lett., 1991, I, 125-128.

[4] [4a] W. Hartwig, U. Schöllkopf, Liebigs Ann. Chem. 1982, 1952-1970. - [4b] U. Schöllkopf, R. Scheuer, Liebigs Ann. Chem. 1984, 939-950.

[5] K. Th. Wanner, A. Kärtner, Synthesis 1988, 968-970.

[6] Nach Einreichen des Manuskripts wurden noch weitere Alkylierungsversuche vorgenommen, die jedoch noch nicht abgeschlossen sind. Bei Alkylierungen mit Ethyliodid wurden gute Ergebnisse erzielt, Umsetzungen mit Isopropyliodid sind bisher noch erfolglos geblieben. Wir danken dem Gutachter für den Hinweis, dies hier noch zu erwähnen.

[7] C. G. Overberger, M. D. Shalati, Eur: Polym, J. 1983, 19, $1055-1065$

[8] D. S. Dunlop, A. Neidle, Anal. Biochem. 1987, 165, 38-44.

[9] W. C. Still, M. Kahn, A. Mitra, J. Org. Chem. 1978, 43, $2923-2925$

$[245 / 92]$ 


\section{Author Index}

Obituary: Kuhn, R. I-XXXI

\begin{tabular}{|c|c|c|c|}
\hline Adam, G., 167 & Dehmlow, E. V., 1159 & Harashima, S., 391, 993 & Kinzinger, L., 877 \\
\hline Aki, S., 83, 97 & Di Stilo, A., 441 & Hartke, K., 1081 & Kirste, B., 897 \\
\hline Albert, A., 801 & Diehl, W., 367 & Hartmann, H., 935 & Kiyota, H., 865, 1201 \\
\hline Albores, M., 231 & Diekmann, H., 1273 & Hartung, H., 1003 & Klimesch, R., 975 \\
\hline Aleksiev, B., 941 & Döhla, B., 231 & Haslinger, E., 413, 751, 959 & Kloc, K., 1239 \\
\hline Amaike, M., 1287 & Dombi, G., 923 & Hassan, A. A., 695 & Klotz, W., 683 \\
\hline Angermund, K., 831 & Drautz, H., 433 & Heinemann, F., 1003 & Knaf, A., 1051 \\
\hline Antus, S., 105, 503, 927 & Drauz, K., 1295 & Helmchen, G., 1313 & Knoll, A., 103 \\
\hline Arsanious, M. H. N., 351 & Drescher, S., 125 & Henkel, G., 1149 & Knoll, K., 777 \\
\hline Asharin-Fard, S., 23 & Dudda, A., 1249 & Hennawy, I. T., 351 & Köditz, J., 1003 \\
\hline Bach, G., 241 & Duddeck, H., 1225 & Henne, P., 565 & Komori, T., 55, 325, 359 \\
\hline Baer, H. H., I & Dulog, L., 201, 691 & Hense, A., 771 & König, W. A., 651 \\
\hline Baitz-Gács, E., 105, 503 & Dutta, D., 1081 & Higuchi, R., 359 & Konopíková, M., 1047 \\
\hline Ballwanz, F., 911 & Eckhardt, E., 255 & Hildebrand, D., 837, 1335 & Köster, R., 189 \\
\hline Baltus, W., 911 & Effenberger, F., 1295, 1303 & Hirsch, H.-L., 231 & Kostuch, A., 1043 \\
\hline Balzarini, J., 513 & Eger, K., 465 & Hofer, O., 99, 355, 595 & Kotzinger, S., 269 \\
\hline Bär, T., 419 & El-Barbary, A. A., 1 & Höffgen, E. C., 531, 543 & Kovaćs, T., 105 \\
\hline Barański, A., 7 & Enders, D., 173, 551 & Hoffmann, B., 333 & Kratz, T., 1149 \\
\hline Barlos, K., 215 & Ermel, K., 1033 & Hoffmann, E., 497 & Kratzer, B., 419 \\
\hline Bartkowski, J.-P. B., 711 & Erndt, A., 1043 & Hoffmann, R. W., 629, 771, & Krause, N., 521 \\
\hline Bats, J. W., 705 & Ernst, L., 1205 & 1185,1193 & Krautstrunk, G., 1069 \\
\hline Baus, U. 207 & Ertl, P., 1047 & Höfle, G., 293, 701, 1017, 1233 & Kreidl, J., 221 \\
\hline Beck-Sickinger, A. G., 1125 & Faillard, H., 485 & Höfner, G., 1273 & Kresze, G., 261 \\
\hline Beckert, R., 11, 1279 & Farkas, M., 221 & Holletz, T., 1051 & Kretzschmar, G., 573 \\
\hline Bedorf, N., 1017 & Farrés, X., 641 & Homann, K., 1155 & Krohn, K., 905,911 \\
\hline Berger, A., 379 & Fechter, M., 379 & Honda, M., 55, 359 & Krotz, A., 1313 \\
\hline Berlin, K., 1153 & Felber, H., 261 & Hopf, M., 1303 & Krüger, C., 831 \\
\hline Bestmann, H. J., 231 & Ferrer, M., 507 & Hosztafi, S., 915 & Kucznierz, R., 189 \\
\hline $\begin{array}{l}\text { Bezergiannidou-Balouctsi, C., } \\
1175\end{array}$ & Fiedorowicz, M., 1043 & Höweler, U., 609 & Kühlwein, J., 1295, 1303 \\
\hline Biermann, U., 645 & Finkam, M.. 551 & Huber-Patz, U., 403, 1133 & Kuhn, N., 1149 \\
\hline Bird, C. W., 1331 & Fišera, L'.. 1047 & Huch, V., 125 & Kula, M.-R., 621 \\
\hline Birk, R., 71 & Flock, M., 451 & Huhn, T., 49, 715 & Kunath, A., 805 \\
\hline Blank, S., 889 & $\begin{array}{l}\text { Fortkamp, J., } 332 \\
\text { Franck B }\end{array}$ & Hußlein, M., 305 & Kunick, C., 1141 \\
\hline Bläser, D., 189 & $\begin{array}{l}\text { Franck, B., } 1069,1075 \\
\text { Freund, S.. } 43\end{array}$ & Hütter, K., 241, 565 & Kunz, M., 975 \\
\hline Blaszczyk, K., 615, 1105 & & Ibrahim, Y. R., 695 & Kurreck, H., 897 \\
\hline Boese, R., 189 & Fruttero, R., 441 & $\begin{array}{l}\text { Iding, M., } 921 \\
\text { Inglot, A D } 1239\end{array}$ & Laatsch, H., 847 \\
\hline Bohnen, H., 1149 & Fuest, M., 629 & $\begin{array}{l}\text { Inglot, A. D., } 1239 \\
\text { Inukai, K., } 359\end{array}$ & $\begin{array}{l}\text { Lackner, H., } 333 \\
\text { Lanzner, W., } 465\end{array}$ \\
\hline Bopp, R., 367 & Fukamatsu, K., 657, 665 & Irngartinger, H., 403, 1133 & Laupichler, L., 343 \\
\hline Bossio, R., 1229 & Fukuda, N., 325, 491 & Irsch, E.-M., 281 & Le thi Quyen, 167 \\
\hline Botyánszki, J., 1225 & Fukuyo, E., 671 & Irschik, H., 293 & Lehmann, J., 1111 \\
\hline Boulos, L. S., 351 & Fürstner, A., 1211 & Isobe, R., 325 & Lehmann, L., 427 \\
\hline Bourauel, T., 711 & Galceran, M., 507 & Jacob, D., 313 & Lichtenthaler, F. W., 967,975 \\
\hline Bracher, F., 837, 1335 & Gangkofner, S., 207 & Janke, F., 179 & Likussar, W., 147 \\
\hline Brader, G., 355 & Gasco, A., 441 & Jansen, J. R., 877 & Lindner, H. J., 61, 1159 \\
\hline Brandstetter, T., 1009 & Gasco, A. M., 441 & Jansen, R., 701 & Link, P., 1161 \\
\hline Braun, H., 261 & Gatos, D., 215 & Jhou, J. X., 359 & Litinas, K. E., 1175 \\
\hline Braun, M., 1091 & Gees, T., 785 & Jiang, Z.-H., 853, 1179 & Lukács, F., 923 \\
\hline Braun, R., 1133 & Gerber, H.-D., 1081 & Jin, B., 809 & Mahler, R., 203 \\
\hline Breiding-Mack, S., 241 & Gerlach, H., 153, 161 & Jonas, J., 1169 & Maier, M. E., 1009 \\
\hline Breitenbücher, J., 201 & Gerth, K., 1017, 1233 & Jørgensen, P. T., I & Malamidou-Xenikaki, E., 1175 \\
\hline Breitmaier, E., 281, 451, 1041, & Gewald, K., 457 & Jończyk, A., 375 & Maletz, R., 1219 \\
\hline 1057,1153 & Giesen, V., 629 & Jung, G., 43, 497, 941, 1125 & Malosse, C., 1201 \\
\hline Bringmann, G., 877 & Giménez, S., 641 & Jung, K.-H., 859 & Mamdapur, V. R., 445 \\
\hline Brockamp, H.-P., 621 & Glassl, B., 583 & Jurkiewicz, E., 565 & Mannila, E., 1037 \\
\hline Brockhausen, I., 721, 737 & Göndös, G., 581 & Kajtár-Peredy, M., 815 & Marcaccini, S., 1229 \\
\hline Bruckmann, J., 831 & Goppelt-Struebe, M., 237 & Kalbacher, H., 131 & Marco, J. A., 99 \\
\hline Cai, M., 141 & Gottsegen. A., 503 & Kalcheva, V., 1319 & Marco, J. L., 801 \\
\hline Camps, P., 641 & Götz, P. H.. 1323 & Kalinowski, H.-O., 1033 & Markowska, A., 1327 \\
\hline Cano, F. H., 801 & Grabley, S., 241, 565, 573 & Kapurniotu, A., 1161 & Marschall, H., 111, 1117 \\
\hline Capuano, L., 125 & Gradnig, G., 379 & Kárpáti, E., 221 & Martin, D., 967 \\
\hline Carganico, G., 641 & Gräf, S., 1091 & Keese, W., 237 & Martin, N., 801 \\
\hline Casas, J., 507 & Graßberger, V., 379 & Keil, D., 935 & Martínez-Grau, A., 801 \\
\hline Cech, D., 1051 & Greger, H., 355 & Keilhofer, D., 947 & Marton, J., 915 \\
\hline Chatterjee, S. K., 437 & Greilich. U., 859 & Keller, M., 871 & Maruoka, H., 625, 1269 \\
\hline Chattopadhyay, S., 445 & Groth, U., 49, 321, 427, 715 & Kellner, M., 847 & Marx, T., 1041 \\
\hline Claßen, A., 183 & Gruner, M., 457 & Kempe, R., 987 & Mauleón, D., 641 \\
\hline Cohen, V. I., 809 & Grzegọzek, M., 823 & Kessel, M., 221 & Maurer, W., 1029 \\
\hline Czibula, L., 221 & Guo, M.. 137 & Kessler, H., 497 & Mayer, M., 573 \\
\hline Dahlhoff, W. V., 831, 1063 & Gusterhuber, D., 147 & Keutel, D., 981 & Meier, H., 313 \\
\hline Dancy, I., 343 & Habermann, A.-K., 987 & Khanbabaee, K., 905 & Meinjohanns, E., 721, 737 \\
\hline Darias, J., 1257 & Hadjieva, P., 1319 & Khlebnikov, V., 77 & Meints, M., 527 \\
\hline Dax, K., 379 & Hammann, P.. 241 & Kido, M., 83, 657, 665 & Meskó, E., 923 \\
\hline De Clercq, E., 513 & Han, X.-B., 853, 1179 & Kimura, T., 325, 491 & Messeguer, A., 507 \\
\hline
\end{tabular}


Metz, K., 55

Metzger, J. O., 203, 645

Meyer, C., 17

Meyer, J.-M., 43

Meyer, W., 1253

Michelitsch, A., 147

Mikhailopulo, I. A., 513

Młochowski, J., 1239

Młotkowska, B., 1327

Mohamed, N. K., 695

Möller, U., 1051

Mori, K., 77, 83, 91, 97, 391, 657 , $665,671,865,993,1201,1287$

Morr, M., 332, 1205

Mourad, A.-F. E., 695

Mueller, M. J., 557

Müller, Alexander, 651

Müller, Andreas, 11, 1279

Müller, V., 975

Nagai, Y., 359

Nagy, J., 815

Nakamura, M., 325

Narjes, F., 841

Neidlein, R., 955

Nemes, A., 221

Nemes, P., 179

Neumann, H.-P., 1313

Neunhoeffer, H., 367, 583

Nicolaides, D. N., 1175

Nieger, M., 451

Nienaber, J., 305

Nowak, W., 153

Nuber, B., 329

Nyitrai, J., 815

Oberhoffner, S., 255

Ogawa, S., 755

Ohkubo, K., 1263

Öhler, E., 269

Olejnik, J., 1327

Orr, J. C., 581

Ortmann, T., 877

Ozegowski, R., 805

Pachaly, P., 281

Paipanova, T., 941

Papaphotiou, G., 215

Para, A., 1043

Paryzek, Z., 615, 1105

Patir, S., 1323

Paulsen, H., 531, 543, 721, 737

Pawar, A. S., 445

Pedersen, E. B., 1

Peng, S., 137, 141

Pepino, R., 1229

Peters, E.-M., 777

Peters, K., 777

Petry, S., 1111

Philipps, S., 573

Piasecki, E., 1239

Pindur, U., 947, 1099

Pink, M., 1145

Plé, N., 583

Porsch, B., 715

Prewo, R., 261

Prónayová, N., 1047

Quast, H., 699, 777

Quéguiner, G., 583

Quermann, R., 1219

Quiñoá, E., 1257
Rainer, H., 117

Rama, N. H., 1331

Reba, R. C., 809

Reck, F., 721, 737

Rehwald, M., 457

Reichardt, C., 23, 935

Reichenbach, H., 1017, 1233

Reichert, H., 403

Reinhardt, R., 1051

Reisch, J., 921

Reischl, W., 587, 595

Reißig, H.-U., 61, 1155, 1159

Renneberg, B., 847

Resch, K., 237

Rettberg, N., 1081

Reuscher, H., 877

Reuther, W., 207

Richter, J., 61, 1159

Richter, L., 427

Richter, N., 49

Riehl, K., 1063

Riguera, R., 1257

Rimpler, M., 237

Ripperger, H., 167

Ritzau, M., 433, 871

Rochat, D., 865, 1201

Röder, E., 711

Rollin, P., 343

Rösel, P., 231

Rosemeyer, H., 513

Roth, A., 485

Rothenhäusler, K., 465

Rotscheidt, K., 1057

Rudorf, W.-D., 1003

Ruland, A., 207

Rümmler, M., 287, 1023

Rustaiyan, A., 111, 1117

Sabatino, P., 441

Saeed, A., 1331

Sahu, D. P., 437

Sajus, H., 211

Samu, J., 1225

Sánchez-Baeza, F., 507

Sander, T., 771, 1185, 1193

Sandhoff, K., 71, 419

San Martín, A., 1257

Sanz-Cervera, J. F., 99

Saul, K., 313

Sażała, M., 1327

Schäfer, G., 23

Schäfer, H. J., 601, 609, 1219

Schäfer, W., 215

Schall, T, 1099

Scharf, H.-D., 117, 183, 447

Schaumann, E., 841

Scheiber, P., 179

Scheick, C., 1245

Scheinkönig, J., 121, 25

Schick, Hans, 103, 805

Schick, Hartmut, 287, 1023

Schilling, G., 287

Schiweck, H., 967

Schmeck, C., 321, 715

Schmid, R. D. 332

Schmidbauer, S. B., 1029

Schmidt, R. R., 71, 419, 683 $853,859,1179$

Schmidtchen, F. P., 261

Schmutterer, H., 1033
Schnaubelt, J., 61, 1159

Schneider, C., 1057

Schneider, G., 923

Schneider, S., 111

Schöllkopf, U., 321, 427, 715

Schomburg, D., 701, 1017

Schönebaum, A., 333

Schönecker, B., 1279

Schoo, N., 601, 609

Schorp, M., 305

Schreiber, K., 167

Schröder, H., 413, 751, 959

Schrötter, E., 103

Schubert-Zsilavecz, M., 147

Schuler, F., 785

Schulze, K., 987

Schummer, D., 293

Schüßler, W., 189

Schwarz, G., 811

Seebach, D., 785, 889

Seela, F., 513

Seligmann, O., 503

Sengupta, S., 437

Seoane, C., 801

Shàmout, A. R., 1159

Shatzmiller, S., 955

Sin, K.-S., 281

Sirges, W., 1075

Snatzke, G., 1225

Sohár, P., 221

Soleymani-Jamarani, M., 705

Somogyi, L., 931

Sorba, G., 441

Spange, S., 98

Spencker, T., 237

Spiteller, G., 17, 121, 251, 1245 , 1249,1253

Stachel, H.-D., 305

Stamenitis, S., 477

Steigel, A., 621

Stelzer, U., 1303

Stephan, H., 43

Stetter, K. O., 871

Stoev, M., 941

Stütz, A. E., 379

Štverková, S., 1169

Syper, L., 1239

Szabó, Z., 915

Szombathelyi, Z., 221

Szpakiewicz, B., 7

Szunyog, J., 105

Szymanowski, M., 103

Takaki, M., 1263

Takayama, S., 91

Takyar, D. K., 651

Talvitie, A., 1037

Teuber, H.-J., 705

Teubner, A., 16

Thiem, J., 211, 343

Thiericke, R., 241, 565, 573

Tiebes, J., 173

Timmermann, R., 1075

Tochtermann, W., 527

Tökés, A. L., 927

Tomioka, H., 671

Tomula, M., 471

Tosheva, M., 1319

Tóth, T. S., 105

Trowitzsch-Kienast, W., 1233
Tsuji, S., 359

Tsunoda, H., 755

Turck, A., 583

Uhlig, H., 987

Uhr, H., 241

Vaaks, E. V., 513

Vasella, A., 261

Vázquez, M. J., 1257

Videnov, G., 941

Visky, G., 221

Voelter, W., 131, 1161

Vollmer, M., 255

von Gersdorff, J., 897

von Schnering, H. G., 777

Vostrowsky, O., 231

Wagner, H., 105, 503

Wahlburg, H.-C., 1117

Wanner, K. T., 477

Watanabe, H., 1287

Weber, L., 987

Weber, T., 967

Weik, C., 955

Weinges, K., 287, 403, 811, 1023 , 1029,1133

Weirich, R., 877

Weiß, D., 11, 1279

Weissensteiner, W., 595

Wessels, P., 871

Weyerstahl, P., 111, 1117

Wild, R., 419

Wink, J., 241, 565, 573

Winkelmann, G., 43

Winterfeldt, E., 137, 141

Witt, S., 65

Witzel, M., 699

Wolff, C., 527

Wolff, J. H., 329

Wolff, J. J., 329

Woźniak, M.. 7, 471, 823

Wray, V., 332, 1233

Wünsch, B., 1273

Wurz, G., 99, 355

Yamagata, K., 625, 1263, 1269

Yamazaki, M., 625, 1263, 1269

Yanagi, K., 671

Yonemitsu, M., 325, 491

Yoshimura, S., 91

Zähner, H., 433

Zaitseva, G. V., 513

Zagar, C., 447

Žák, Z., 1169

Zdrojewski, T., 375

Zeeck, A., 241, 433, 565, 573, 871

Zenk, M. H., 557

Zhang, L., 141

Zhang, P., 35

Zhang, X.-s., 35

Zhao, C., 35

Ziegler, H. J., 1029

Ziegler, M. L., 1313

Ziegler, T., 255

Zierer, D., 691

Zimmer, R., 1155

Zimmer, S., 497

Zimmermann, P., 859

Zimmermann, T., 1145

Zoukas, T., 305

Zschiesche, R., 61, 1159

Zugenmaier, P., 1063 